\title{
The effects of spatial resolution on integral field spectrograph surveys at different redshifts - The CALIFA perspective
}

\author{
D. Mast ${ }^{1,2}$, F. F. Rosales-Ortega ${ }^{3,4}$, S. F. Sánchez ${ }^{2,1}$, J. M. Vílchez ${ }^{2}$, J. Iglesias-Paramo ${ }^{2,1}$, C. J. Walcher ${ }^{5}$, \\ B. Husemann ${ }^{5}$, I. Márquez ${ }^{2}$, R. A. Marino ${ }^{6}$, R. C. Kennicutt ${ }^{7}$, A. Monreal-Ibero ${ }^{2,5}$, L. Galbany ${ }^{8}$, \\ A. de Lorenzo-Cáceres ${ }^{9}$, J. Mendez-Abreu ${ }^{10,11}$, C. Kehrig ${ }^{2}$, A. del Olmo ${ }^{2}$, M. Relaño ${ }^{12}$, L. Wisotzki ${ }^{5}$, \\ E. Mármol-Queraltó ${ }^{10,11}$, S. Bekeraitè ${ }^{5}$, P. Papaderos ${ }^{13}$, V. Wild ${ }^{9}$, J. A. L. Aguerri ${ }^{10,11}$, J. Falcón-Barroso ${ }^{10,11}$, \\ D. J. Bomans ${ }^{14,15}$, B. Ziegler ${ }^{16}$, B. García-Lorenzo ${ }^{10,11}$, J. Bland-Hawthorn ${ }^{17}$, \\ Á. R. López-Sánchez ${ }^{18,19}$, and G. van de Ven ${ }^{20}$ \\ (Affiliations can be found after the references)
}

Received 29 April 2013 / Accepted 15 November 2013

\begin{abstract}
Context. Over the past decade, 3D optical spectroscopy has become the preferred tool for understanding the properties of galaxies and is now increasingly used to carry out galaxy surveys. Low redshift surveys include SAURON, DiskMass, ATLAS3D, PINGS, and VENGA. At redshifts above 0.7 , surveys such as MASSIV, SINS, GLACE, and IMAGES have targeted the most luminous galaxies to study mainly their kinematic properties. The on-going CALIFA survey $(z \sim 0.02)$ is the first of a series of upcoming integral field spectroscopy (IFS) surveys with large samples representative of the entire population of galaxies. Others include SAMI and MaNGA at lower redshift and the upcoming KMOS surveys at higher redshift. Given the importance of spatial scales in IFS surveys, the study of the effects of spatial resolution on the recovered parameters becomes important.

Aims. We explore the capability of the CALIFA survey and a hypothetical higher redshift survey to reproduce the properties of a sample of objects observed with better spatial resolution at lower redshift.

Methods. Using a sample of PINGS galaxies, we simulated observations at different redshifts. We then studied the behaviour of different parameters as the spatial resolution degrades with increasing redshift.

Results. We show that at the CALIFA resolution, we are able to measure and map common observables in a galaxy study: the number and distribution of $\mathrm{H}$ II regions ( $\mathrm{H} \alpha$ flux structure), the gas metallicity (using the $\mathrm{O} 3 \mathrm{~N} 2$ method), the gas ionization properties (through the [N II]/H $\alpha$ and $[\mathrm{O} \mathrm{III}] / \mathrm{H} \beta$ line ratios), and the age of the underlying stellar population (using the $\mathrm{D}_{4000}$ index). This supports the aim of the survey to characterise the observable properties of galaxies in the Local Universe. Our analysis of simulated IFS data cubes at higher redshifts highlights the importance of the projected spatial scale per spaxel as the most important figure of merit in the design of an integral field survey.
\end{abstract}

Key words. techniques: spectroscopic - galaxies: abundances - stars: formation - galaxies: ISM - galaxies: stellar content

\section{Introduction}

The Calar Alto Legacy Integral Field Area Survey (CALIFA, $0.005<z<0.03$; Sánchez et al. 2012a) aims to characterise spectroscopically the galaxy population in the Local Universe, and on completion will be the largest and most comprehensive wide-field integral field spectrograph (IFS) survey carried out to date. With its statistically significant sample of $\sim 600$ galaxies, CALIFA will form the bridge between large single aperture surveys and detailed studies of individual galaxies.

Other surveys in the Local Universe using the power of integral-field spectrophotometers for a detailed study of nearby galaxies include the SAURON (Spectroscopic Areal Unit for Research on Optical Nebulae) project (de Zeeuw et al. 2002) and its extension ATLAS3D $(z<0.01$; Cappellari et al. 2011), VENGA (VIRUS-P Exploration of Nearby Galaxies, Blanc et al. 2010), the DiskMass Survey (Bershady et al. 2010), and the PPAK IFS Nearby Galaxies Survey, PINGS $(z \sim 0.002$; Rosales-Ortega et al. 2010, hereafter RO10). Upcoming surveys are SAMI (Sydney Australian Astronomical Observatory Multi-object Integral Field Spectrograph, Croom et al. 2012) and $\mathrm{MaNGA}^{1}$ (Mapping Nearby Galaxies at APO, $z \sim 0.05$ ).

\footnotetext{
1 http://www.sdss3.org/future/manga.php
}

At high redshift there is the SINS (Spectroscopic Imaging survey in the Near-infrared with SINFONI) survey $(z \sim 2$; Förster Schreiber 2009), the MASSIV (Mass Assembly Survey with SINFONI in VVDS) survey $(z \sim 1$; Vergani et al. 2012), the GLACE (GaLAxy Cluster Evolution) survey $(z \sim 0.6$; Sánchez-Portal et al. 2011), and the IMAGES (MAss Galaxy Evolution Sequence; $z \sim 1$; Ravikumar et al. 2007).

In order to map the properties of local galaxies, a compromise must be made between the observing time invested on each object and the number of objects of the sample. The mosaicking approach designed for the PINGS survey, which mapped the H II regions over the whole extension of the galaxies and explored the 2D metallicity structure of discs, involved more than 30 pointings and three years of observation. In contrast, the SAURON project opted for single pointings of the central part of early-type galaxies and bulges of spirals.

The CALIFA survey was designed with the aim of obtaining a balance between a detailed study of each galaxy and a statistically significant sample to fully characterise the optical properties of galaxies in the Local Universe. With this in mind, the sample was selected to have optical sizes that fit within the field of view (FoV) of the PPak instrument (PMAS - Potsdam MultiAperture Spectrophotometer - fiber pack, Roth et al. 2005; Verheijen et al. 2004; Kelz et al. 2006; Kelz \& Roth 2006), 

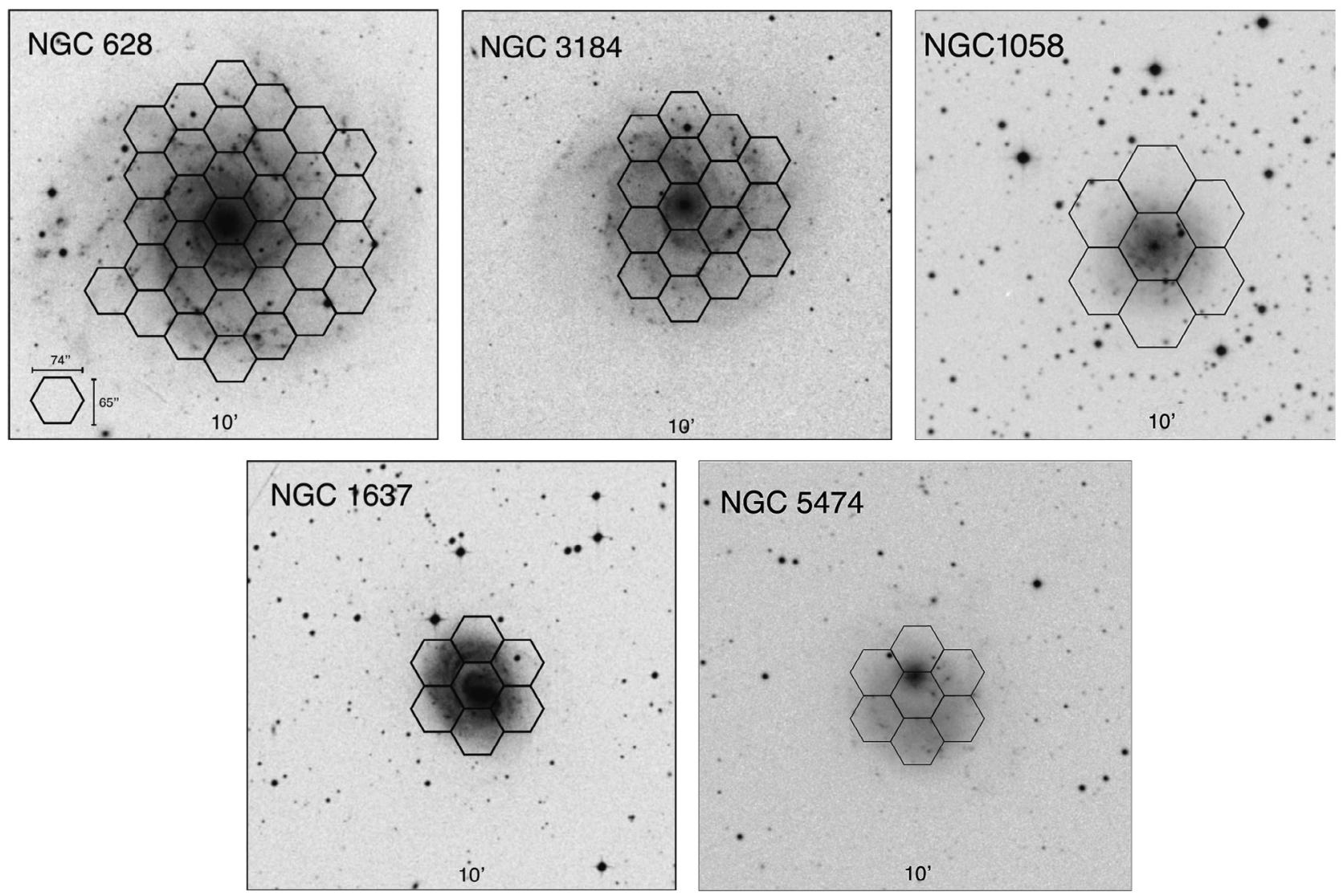

Fig. 1. Digital Sky Survey $R$-band images of the five galaxies selected from PINGS for this study (adapted from RO10). The IFS mosaicking is indicated by the superposition of the hexagonal PPak FoVs. Images are $10 \times 10 \mathrm{arcmin}^{2}$. North is up, east is to the left.

allowing us to map their physical properties over their whole optical extent in just one pointing. In this context, one of the aims of the present paper is to determine the capability of CALIFA to measure the main properties of the observed galaxies despite resolution limitations.

Looking ahead to the next decade when new proposed surveys will explore the intermediate and high redshift regime, in this paper we also study how the information loss due to spatial resolution degradation will affect an hypothetical IFS survey at higher redshift. Taking advantage of the superb spatial sampling of a subsample of PINGS galaxies $(z \sim 0.0009-0.0023$, $\approx 0.04 \mathrm{kpc} / \mathrm{arcsec}$ ), we create two simulations: simulation $Z 1$ represents CALIFA at $z \sim 0.02$, and simulation $Z 2$ represents an hypothetical survey at $z \sim 0.05$, or other redshift ranges depending on the instrument. In what follows, we refer to the PINGS redshift regime at an average $z \sim 0.002$ as Z0. We will analyse the behaviour of some common observables used in galaxy studies as the spatial resolution degrades with increasing redshift. Because of the low spectral resolution of PINGS data, we are unable to study the effect of spatial resolution on kinematics because the simulated cubes would not be representative of the higher spectral resolution CALIFA data.

Previous studies of this issue include Viironen et al. (2012) with a detailed study of UGC $9837(z \sim 0.008863)$ repeating the analysis with simulated versions of the galaxy at higher redshifts $(z \approx 1-2)$. Kronberger et al. (2007) model disc galaxies using $N$-body/hydrodynamic simulations to investigate distortions in the velocity fields at different redshifts from $z=0$ to $z=1$. Giavalisco et al. (1996) simulate Hubble Space Telescope (HST) images at cosmological distances using images from the Ultraviolet Imaging Telescope (UIT), or the more recent work of Barden et al. (2008) where they simulate galaxies at redshifts $0.1<z<1.1$ using images from the SDSS in the $u, g, r, i$, and $z$ filter bands as input. Most of these studies have focussed on the degradation of images with redshift. Examples of studies focussing on spectral effects are Law et al. (2006) who mapped the velocity fields of high-redshift galaxies, and Yuan et al. (2013) who utilised the $[\mathrm{N} \mathrm{II}] / \mathrm{H} \alpha$ ratio to investigate how an inferred metallicity gradient is altered by the loss of angular resolution.

The structure of this paper is as follows. In Sect. 2 we present the sample. Section 3 summarises the observations and data reduction while Sect. 4 explains how we simulate the different redshift regimes. The analysis of both gaseous and stellar components of the sample is presented in Sect. 5. In Sect. 6 we review the main results of this work.

Throughout this paper, we assume a standard $\Lambda$ CDM cosmology consistent with WMAP results (Bennett et al. 2003) with $\Omega_{\mathrm{m}}=0.3, \Omega_{A}=0.7$ and $H_{0}=70 h_{70} \mathrm{~km}^{-1} \mathrm{~s}^{-1}$.

\section{Sample}

The PINGS survey is a project designed to construct 2D spectroscopic mosaics of a representative sample of 17 nearby spiral galaxies, using PMAS in the PPak mode at the Centro Astronómico Hispano Alemán (CAHA) at Calar Alto, Spain. PINGS is one of the most detailed and largest IFS surveys of individual galaxies at low redshift $(z \sim 0.001)$. The data cover most of the optical extent of the galaxies, down to $R_{25}$ (the radius of the galaxy at the isophotal level of $25 \mathrm{mag} / \mathrm{arcsec}^{2}$ in $B$-band), with one of the best spatial resolutions and spectroscopic coverage achieved so far in any IFS survey. From the PINGS sample, we selected the five galaxies (Fig. 1) with the largest angular 
D. Mast et al.: The effects of spatial resolution on integral field spectrograph surveys at different redshifts

Table 1. PINGS subsample.

\begin{tabular}{lcccc}
\hline \hline Object & Type & Dist. [Mpc] & Size $\left[\operatorname{arcmin}^{2}\right]^{a}$ & $z$ \\
\hline NGC 628 & SA(s)c & 9.3 & $10.5 \times 9.5$ & 0.00219 \\
NGC 1058 & SA(rs)c & 10.6 & $3.0 \times 2.8$ & 0.001728 \\
NGC 1637 & SAB(rs)c & 12.0 & $4.0 \times 3.2$ & 0.00239 \\
NGC 3184 & SAB(rs)cd & 11.1 & $7.4 \times 6.9$ & 0.001975 \\
NGC 5474 & SA(s)cd & 6.8 & $4.8 \times 4.3$ & 0.000911 \\
\hline
\end{tabular}

Notes. ${ }^{(a)}$ Size of the galaxy down to $\mathrm{R}_{25}$ (de Vaucouleurs et al. 1992).

size (see Table 1 for details of each galaxy). The NGC 628 mosaic subtends $34 \operatorname{arcmin}^{2}$ and was constructed from 34 different pointings, the NGC 1058 mosaic subtends $3.0 \times 2.8 \mathrm{arcmin}^{2}$ and was constructed from 7 different fields, the NGC 1637 mosaic was built from one central pointing and a concentric ring of 6 pointings covering a total area of $4.0 \times 3.2 \mathrm{arcmin}^{2}$, the NGC 3184 mosaic covers an area of $7.4 \times 6.9 \mathrm{arcmin}^{2}$ and was constructed from 16 IFS pointings, and finally the NGC 5474 mosaic covers an area of $4.8 \times 4.3 \mathrm{arcmin}^{2}$ and was built from of one central pointing and a concentric ring of 6 pointings.

\section{Observations and data reduction}

The observations were carried out at the $3.5 \mathrm{~m}$ telescope of the Calar Alto observatory with the PMAS in the PPak mode. The PPak unit features a central hexagonal bundle with 331 densely packed optical fibres to sample an astronomical object with a resolution of $2.7^{\prime \prime} /$ fibre, over an area of $74 \times 65 \operatorname{arcsec}^{2}$, with a filling factor of $65 \%(<100 \%$ due to gaps in-between the fibres). The sky background is sampled by 36 additional fibres distributed in 6 mini-bundles of 6 fibres each, which encircle the central hexagon at a distance of $\sim 90^{\prime \prime}$. All galaxies were observed using the same telescope and instrument setup. The V300 grating was used, covering a wavelength range of 3700-7100 $\AA$ with a resolution of $\sim 10 \AA$ FWHM, corresponding to $\sim 600 \mathrm{~km} \mathrm{~s}^{-1}$. The final product of the data reduction is a set of row-staked-spectra (RSS), a 2D FITS image where the $X$ and $Y$ axes contain the spectral and spatial information respectively, regardless of their position in the sky. An additional file links the different spatial elements to position on the sky. Row-staked-spectra present a discontinuous sampling of the sky, so we performed an interpolation to obtain a regularly sampled 3D cube (datacube). Not all PINGS datacubes have the same final sampling. NGC 1637, NGC 1058, NGC 3184 and NGC 5474 have $1^{\prime \prime} /$ spaxel sampling, while NGC 628 has a sampling of $2 "$ /spaxel. For more details on the observing strategy, data reduction (similar to that used for the CALIFA data) and sample selection of the PINGS survey, see RO10.

\section{Methodology}

The aim of this work is to study how the loss of spatial resolution due to redshift affects the derivation of physical parameters obtained from IFS observations at different redshifts. We study three different spatial scales: (i) $Z 0$ is at the original resolution of the PINGS data set, i.e. sampling of $\sim 0.045 \mathrm{kpc} /{ }^{\prime \prime}$, or 80-100 spatial elements across the optical extent; (ii) $Z 1$ is at the typical spatial resolution and sampling of a CALIFA galaxy of diameter $\sim 60^{\prime \prime}$, with $25-30$ spatial elements out to $\sim 2-3$ effective radii ( $\mathrm{Re}$ ); (iii) $Z 2$ corresponds to a galaxy sampled
Table 2. Spatial sampling at $Z 0, Z 1$ and $Z 2$.

\begin{tabular}{lccc}
\hline \hline \multicolumn{4}{c}{ NGC 628 [2"/pixel] } \\
\hline$z$ & 0.00219 & 0.0135 & 0.056 \\
pixel [kpc/pix.] & 0.09 & 0.27 & 1.08 \\
\hline \multicolumn{4}{c}{ NGC $1637\left[1^{\prime \prime} /\right.$ pixel] } \\
\hline$z$ & 0.002392 & 0.007 & 0.028 \\
pixel [kpc/pix.] & 0.05 & 0.144 & 0.562 \\
\hline \multicolumn{4}{c}{ NGC $1058\left[1^{\prime \prime} /\right.$ pixel] } \\
\hline$z$ & 0.001728 & 0.005 & 0.02 \\
pixel [kpc/pix.] & 0.036 & 0.103 & 0.405 \\
\hline \multicolumn{4}{c}{ NGC 3184 [1"/pixel] } \\
\hline$z$ & 0.001975 & 0.004 & 0.015 \\
pixel [kpc/pix.] & 0.041 & 0.083 & 0.306 \\
\hline \multicolumn{4}{c}{ NGC 5474 [1"/pixel] } \\
\hline$z$ & 0.000911 & 0.003 & 0.01 \\
pixel [kpc/pix.] & 0.019 & 0.062 & 0.205 \\
\hline \multicolumn{4}{c}{}
\end{tabular}

at $1 \mathrm{kpc} /{ }^{\prime \prime}$ or $\sim 7-10$ spatial elements out to $\sim 2-3$ Re i.e. a $z \sim 0.05$ galaxy and instrument with $\sim 1^{\prime \prime}$ spatial elements. We should note that the key parameter is the number of spatial elements across the extent of the galaxy, the fiducial redshift of the galaxy will depend on the precise survey design. We then performed the same analysis on the three sets of galaxies (i.e. the original PINGS galaxies and both simulated sets) focusing on a set of commonly measured observables. In this way, we can quantify the extent to which we can recover observables of interest, and thus achieve the aim of characterising the properties of galaxies in the Local Universe, in both CALIFA and a hypothetical higher redshift IFS survey.

We decided to focus this study only on resolution effects, and in particular on spatial resolution degradation due to redshift. We have not considered either surface brightness dimming or increase in noise, i.e. we take for granted that the exposure time is sufficient to reach a similar depth in all cases. Also, we have not taken into account the effects of PSF, since, so far, the ability to spatially resolve a feature in an IFS dataset is more strongly affected by the sampling than by PSF size.

\subsection{Simulations}

We binned the PINGS cubes using R3D to match the Z1 (CALIFA) sampling, and then binned again these cubes to match the $Z 2$ sampling. Given an original cube of size $N_{x} \times N_{y}$ and a bin factor $f$, we add all the spectra in a box of size $f \times f$ and generate a new cube of size $N_{x} / f \times N_{y} / f$. The spatial sampling detailed above results in $f=3$ and $f=12$ for all galaxies except for NGC 3184 where we used $f=2$ and $f=8$. As we are not considering noise, nor cosmological dimming, we consider the mean flux of the binned pixels, rather than integrating. In the absence of noise this will be statistically identical, and makes direct comparisons much easier. Table 2 gives the spatial sampling and fiducial redshift for each galaxy in the three surveys. To avoid binning artefacts such as border discontinuities, we applied a 2D Gaussian smoothing to the cubes after binning. This resembles the effects of an interpolation kernel passed over fibre-based (dithered or not) IFS observations. We have also tested convolution of the data before binning, more appropriate for regularly gridded observations, such as SINFONI, VIMOS, MUSE or GMOS. In all tests we obtained qualitatively similar, although quantitatively different, results. The differences do not affect the conclusions of this work in any way. Figure 2 shows 

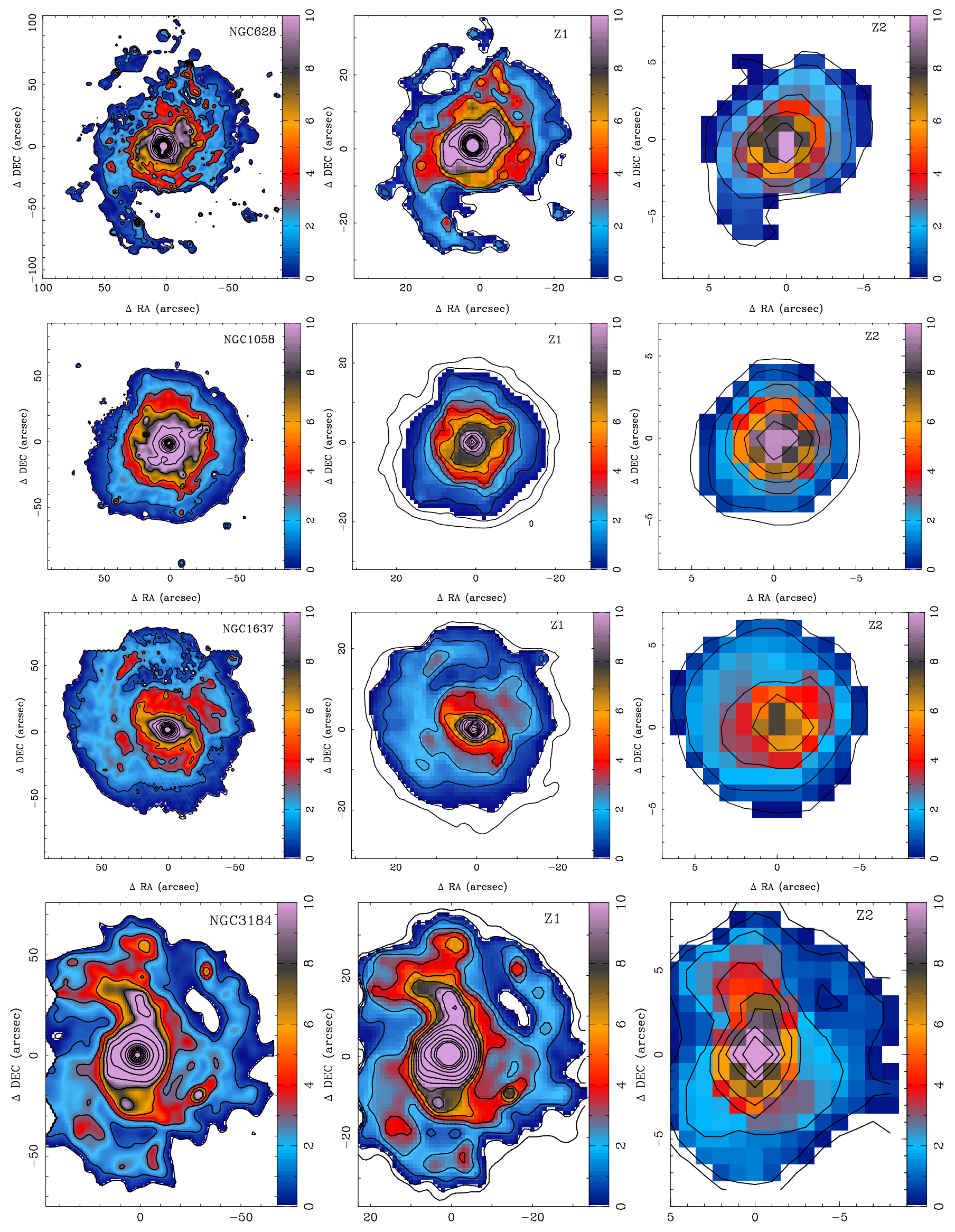

$\triangle \mathrm{RA}(\operatorname{arcsec})$

$\triangle \mathrm{RA}(\operatorname{arcsec})$

$\triangle \mathrm{RA}(\operatorname{arcsec})$

Fig. 2. $V$-band images constructed from the datacubes. Flux scale in arbitrary units. Left: original PINGS galaxies. Middle: Z1 galaxies. Right: $\mathrm{Z2}$ galaxies. North is up, east is to the left. 

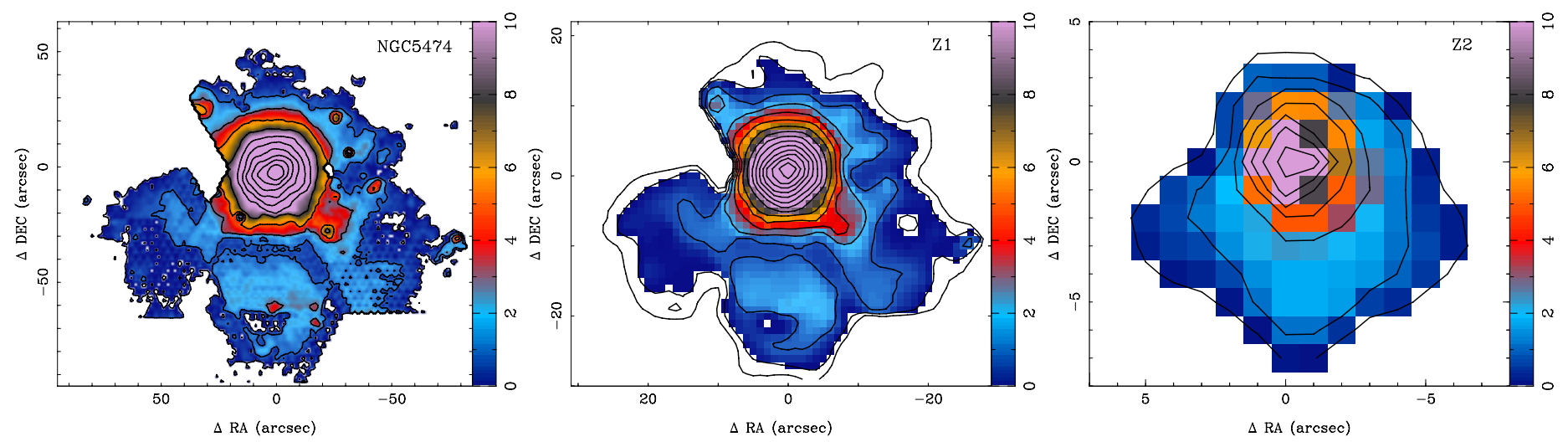

Fig. 2. continued.

$V$-band images of the original and simulated galaxies. The images are constructed by convolving a Johnson $V$-band filter with the datacubes.

\section{Analysis}

The aim of this paper is to understand how observed properties of $\mathrm{H}$ II regions, in particular radial trends and spatial distribution, are affected by spatial resolution degradation. A complete analysis of the behaviour of derived properties and impact on physical conclusions will be the subject of forthcoming papers.

We performed a set of standard analyses of increasing complexity: (i) reconstruction of broad-band images from the IFS datacubes; (ii) creation of emission line flux maps; (iii) measurement of more complex spectroscopic properties, such as line ratios, chemical abundance tracers, and ionisation indicators, following the procedures described in Sánchez et al. (2012a,b).

To analyse the information contained in the cubes, we used FIT3D to separate the underlying stellar continuum from the emission lines in each spectrum, following the process described in detail in Sánchez et al. (2011) and RO10. FIT3D routines fit the underlying stellar population combining linearly a set of stellar templates within a multi-SSP model. A simple SSP template grid was adopted, consisting of three ages $(0.09,1.00$ and $17.78 \mathrm{Gyr})$ and two metallicities $(Z \sim 0.0004$ and 0.03$)$. The SSP templates were taken from the MILES project (Vazdekis et al. 2010). The metallicities and ages cover the wide ranges possible within the MILES library. The oldest stellar population was selected to reproduce the reddest possible underlying stellar population, mostly due to higher metallicities than the one considered in our simplified model, although it is clearly older than the accepted cosmological age of the Universe. Our youngest stellar population is the 2nd youngest in the MILES library for which both metallicities are available. No appreciable difference was found between using this or the youngest SSP ( $~ 80 \mathrm{Myr})$. Finally, we selected an average age stellar population, of $\sim 1 \mathrm{Gyr}$, required to reproduce the intermediate-to-blue stellar populations, and to produce more reliable corrections of the underlying stellar absorption.

We study commonly used indicators of gaseous and stellar properties. In the case of the gas emission, these are the $\mathrm{H} \alpha$ flux, the [O III] $\lambda 5007 / \mathrm{H} \beta$ vs. [N II] $\lambda 6583 / \mathrm{H} \alpha$ BPT diagnostic diagram (Baldwin et al. 1981; Veilleux \& Osterbrock 1987), and the radial oxygen abundance gradient derived from the widely-used O3N2 strong-line indicator (Pettini \& Pagel 2004; Marino et al. 2013). For the stellar component we decided to show the $4000 \AA$ break $\mathrm{D}_{4000}$ (i.e. the break strength at $4000 \AA$ defined as the ratio of the average flux densities in the narrow bands 4000-4100 $\AA$ and 3850-3950 $\AA$, Balogh et al. 1999) as it is the most commonly used proxy for mean stellar age.

All radial profiles are plotted in units of $R_{25}$ obtained from the RC3 catalogue (de Vaucouleurs et al. 1992) and scaled to each redshift regime. Surface brightness profiles for each galaxy (Fig. 3) were constructed using the IRAF ${ }^{2}$ task ellipse and then scaled in the $y$-axis to make them coincide at $R_{\max } / 2$ (maximum measured radius divided by 2 ), in order to analyse the profile differences between galaxies. For the ellipse fitting we used $V$-band images extracted from the datacubes as explained before.

All the above analyses (fitting and subtracting the underlying stellar continuum and fitting of the emission lines) were performed independently on each datacube, i.e both the original and simulated ones.

\subsection{Morphology}

From Fig. 2 we can see that at the representative redshift of CALIFA ( $Z 1$, middle panel) we are able to qualitatively reproduce most morphological features. In all cases we can trace the spiral arms, rings and bulge extension seen in the original images. Finer detail such as individual regions or spots, are smeared out on the $Z 1$ case, so qualitatively we can see that the analysis of the distribution of some features along the arms or small regions will be limited by the resolution effect.

In the $Z 2$ regime (Fig. 2, right panel), the situation is totally different. Except in the case of NGC 628, where, as a granddesign galaxy, the spiral arms are very well defined and still traceable to high redshift, in all other galaxies the identification of spiral signatures is hampered. Individual regions are not easily detected and the separation between the bulge and the disc becomes subtle. The latter effect can also be appreciated from the surface brightness profiles in Fig. 3. In all cases, the primary effect of spatial resolution loss is that the profiles become flatter in the centre. In the $Z 1$ case the central component, though smoothed, can be detected as an excess of light above the inward extrapolation of the disc. That is not the case for the $Z 2$ profiles where the inner regions are shallow and a single component fit to the data seems to be the best option, although a slight disc/bulge differentiation could be seen in the radial plots.

2 IRAF is distributed by the National Optical Astronomy Observatories, which are operated by the Association of Universities for Research in Astronomy, Inc., under cooperative agreement with the National Science Foundation. 

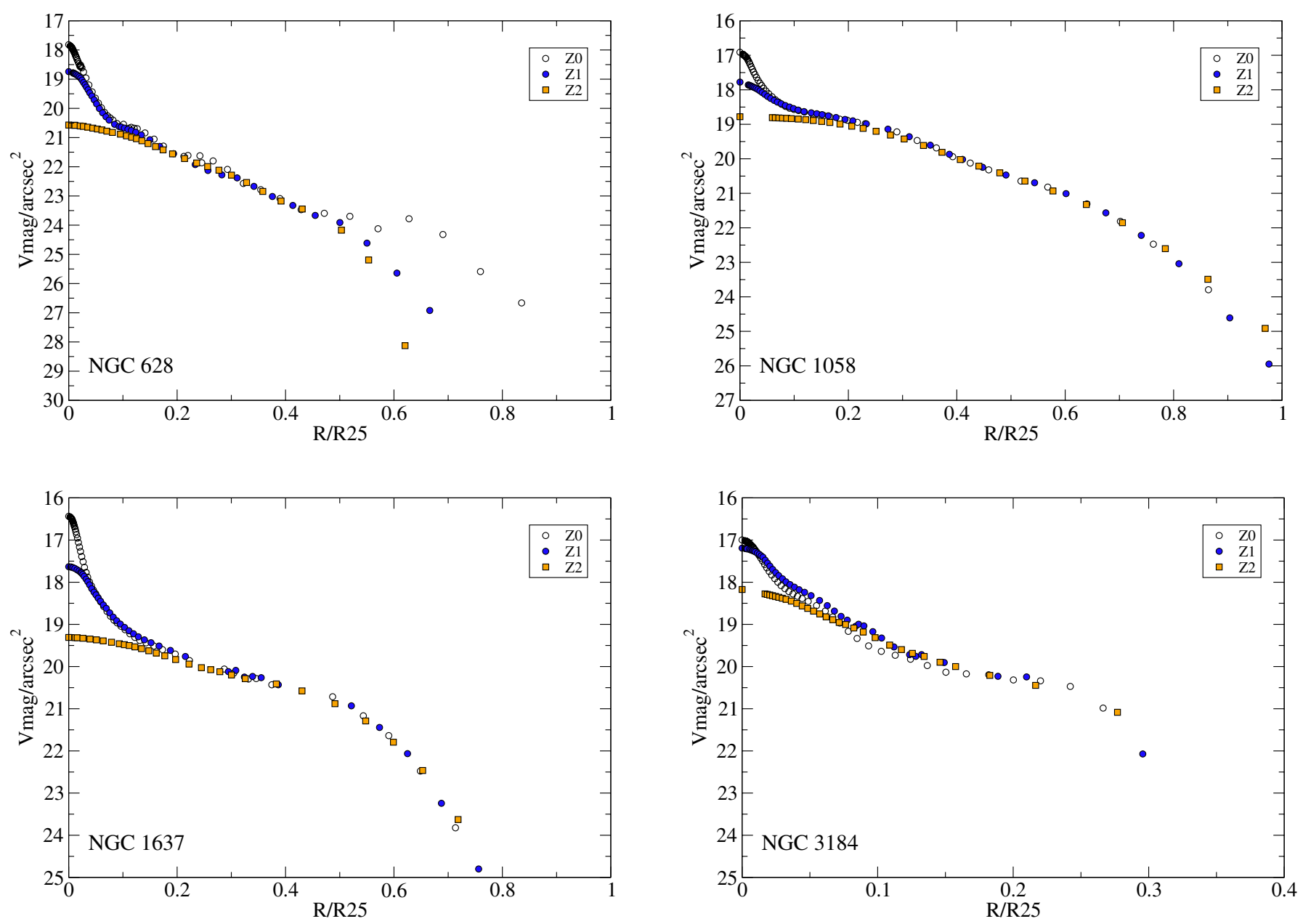

Fig. 3. Surface brightness profiles of the original PINGS galaxies and the simulated ones, constructed fitting ellipses to $V$-band images extracted from the datacubes. The profiles were scaled in the $y$-axis to make them coincide at $R_{\max } / 2$ (maximum measured radius divided by 2 ).

\subsection{Ionized gas component}

\subsubsection{H II region distribution}

To extract the $\mathrm{H} \alpha$ intensity maps for all the cubes (see Figs. 4 and 5) we co-add the flux intensity within a square-shaped simulated filter centred at the wavelength of $\mathrm{H} \alpha(6563 \AA)$ shifted to the observed frame considering the redshift of the object, with a width of $60 \AA$. The adjacent continuum for each pixel was estimated averaging the flux intensity within two bands on both sides of the centre, separated $100 \AA$ from it and with a width of $60 \AA$. This continuum intensity is then subtracted from the $\mathrm{H} \alpha$ intensity to derive a continuum-subtracted emission line map. It is worth noting that this $\mathrm{H} \alpha$ intensity map is contaminated with the adjacent [N II] emission lines, and it is not corrected for Balmer absorption of the underlying stellar population.

Figure 4 show the resulting maps from the original and degraded datacubes. The $Z 1$ maps show the same distribution of $\mathrm{H}$ II regions tracing the spiral arms as in the $Z 0$ case. Because of the coarse resolution, the $\mathrm{H}$ II regions found in the $Z 1$ case are complexes of several PINGS regions but without a severe alteration of $\mathrm{H} \alpha$ flux local maxima position. On the contrary, as can be expected from the global morphology noted in the previous section, the $\mathrm{H} \alpha 2 \mathrm{D}$ distribution in the $\mathrm{Z} 2$ simulation, presents a strong difference as many $\mathrm{H}$ II complexes are averaged into one single $\mathrm{H} \alpha$ aggregation. Again, no traceable spiral arms are visible for the Z2 regime, and even in the NGC 628 case, in which the spiral structure is perfectly traced by the $\mathrm{H}$ II complexes at $Z 0$ and $Z 1$, the $Z 2$ galaxy presents a morphology that at a first sight it is not easily associated with a spiral structure. In any case, it is worth noting that the morphological analysis presented here is meant to show the effects of resolution in the morphology, in the understanding that any spectroscopic survey will have an imaging follow up to derive the morphology with better resolution and that kind of work will not be done from the line maps (although this might not be the case for all high-redshift studies, since some of them relies only in the IFS data).

We used the HIIEXPLORER software (Sánchez et al. 2012b) to segregate and perform the corresponding spectral extraction of all the H II regions detected on each map. The HIIEXPLORER works assuming: (a) H II regions are peaky/isolated structures with strong ionized gas emission clearly above the continuum level emission and the average ionized gas emission across the galaxy, and (b) H II regions have a typical physical size of about a hundred or few hundreds of parsecs (e.g. González Delgado \& Pérez 1997; López et al. 2011; Oey et al. 2003), which corresponds to a typical projected size of a few arcsec at the distance of the observed galaxies. The algorithm requires a line emission map, with the same world-coordinate system (WCS) and resolution as the input datacube (preferentially an $\mathrm{H} \alpha$ emission line map). HIIEXPLORER outputs a segmentation FITS file 
D. Mast et al.: The effects of spatial resolution on integral field spectrograph surveys at different redshifts
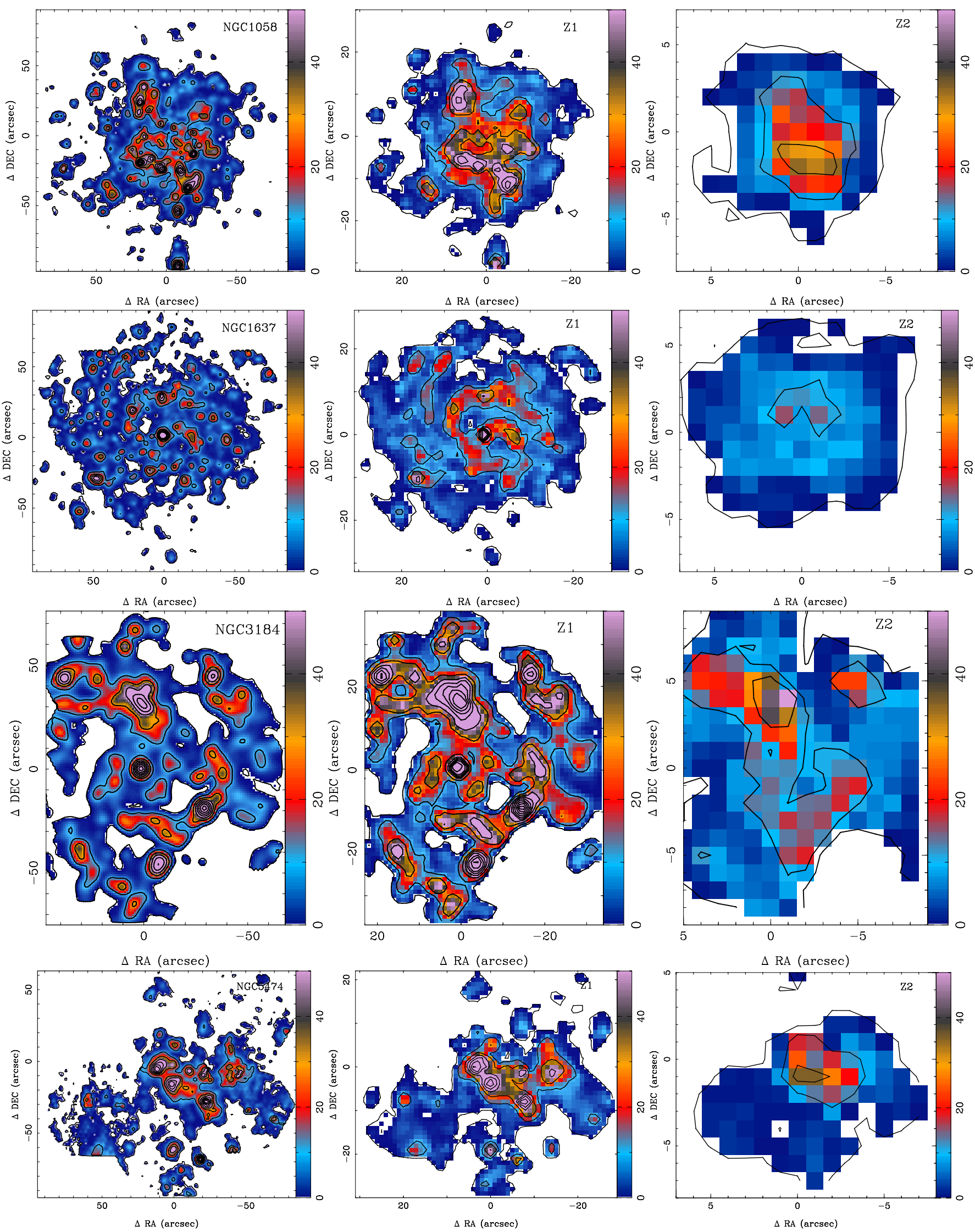

Fig. 4. $\mathrm{H} \alpha$ images extracted from the cubes. Flux is in units of $10^{-16} \mathrm{erg} \mathrm{s}^{-1} \mathrm{~cm}^{-2} \AA^{-1}$. $Z 1$ and $Z 2$ are scaled to $Z 0$ flux. Left: original $Z 0$ galaxies. Middle: $Z 1$ galaxies. Right: $Z 2$ galaxies. North is up, east is to the left. The H $\alpha$ map of NGC 628 is displayed in Fig. 5. 

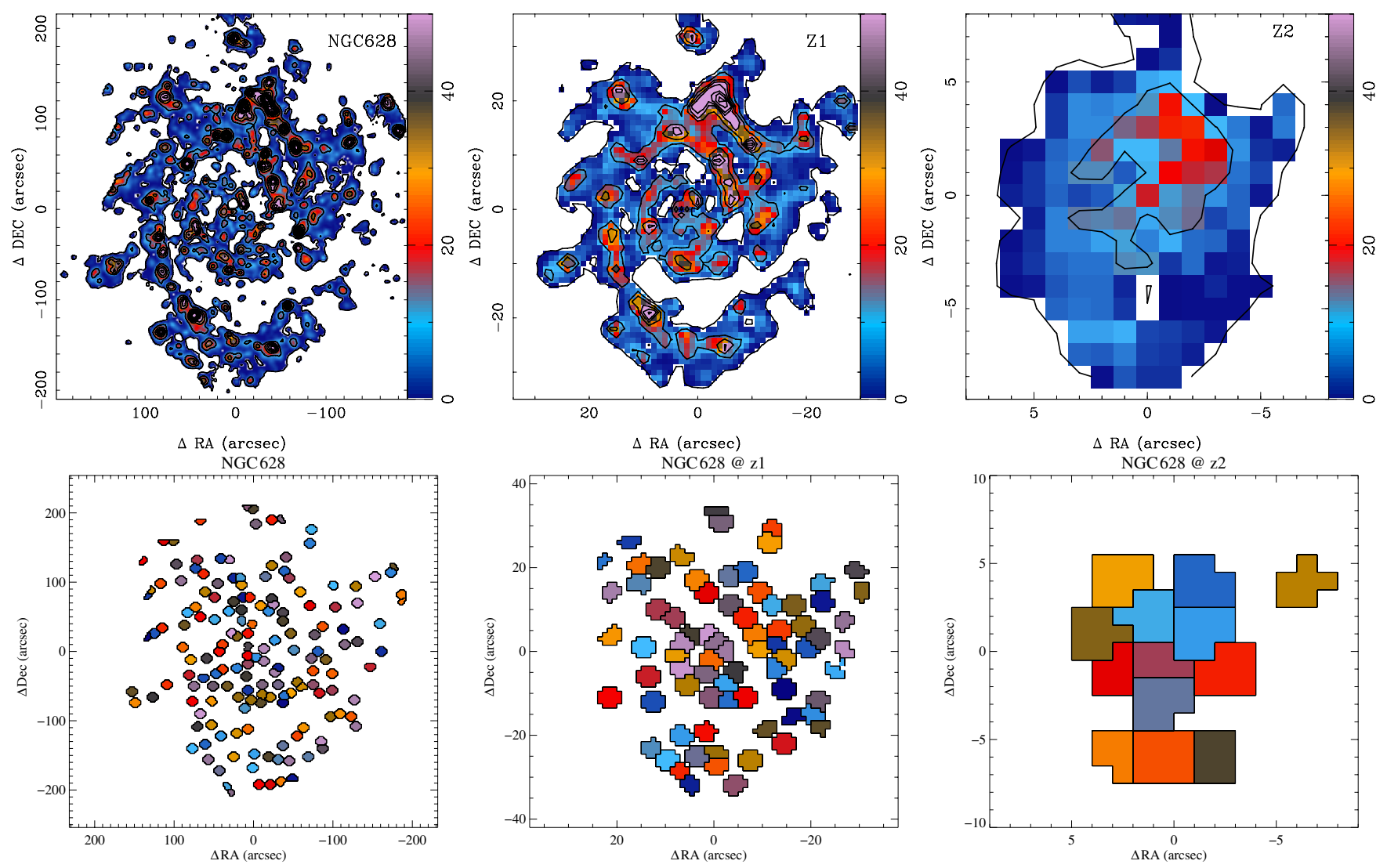

Fig. 5. H II regions detection showing the application of the HIIEXPLORER software to NGC 628. The panels display the segmentation maps generated by the software. Left: original Z0 galaxies. Middle: Z1 galaxies. Right: Z2 galaxies. North is up, east is to the left. These segmentation maps are later used for the extraction of the $\mathrm{H}$ II region spectra.

describing the pixels associated to each $\mathrm{H}$ II region map that should be used for the extraction of the spectra. Each region will have a single spectrum generated by co-adding all the spectra indicated by the segmentation map. In Fig. 5 we can see, as an example, the case of NGC 628 in the three redshift regimes. The number of $\mathrm{HII}$ regions detected in each regime immediately indicates the effect of resolution degradation, i.e. as we go to higher redshift we are considering as one region the contribution from all $\mathrm{HII}$ regions smaller than the resolution element. Even in the $Z 0$ case, the real physical size of an $\mathrm{H}$ II region is below the spatial resolution: in all cases, when we refer to $\mathrm{H}$ II regions we are in fact considering H II complexes or aggregates. Table 3 shows the number of detected regions, with good enough signal-to-noise ratio $(\mathrm{S} / \mathrm{N})$ to perform the analysis, following the criteria from Sánchez et al. (2012b), for all the galaxies in the present study. For example, from 331 regions detected in NGC 1058, the Z2 regime identifies only 7 . For the $Z 1$ case, the average factor of under-detection is $\sim 3$, and the difference between $Z 1$ and the $Z 2$ regime $\sim 5$. Also, as the resolution becomes coarser, the difficulty in delimiting the $\mathrm{H}$ II regions implies that we are combining both diffuse gas and $\mathrm{H}$ II region emission. It is important to mention that this is always the case if one is considering spaxel-to-spaxel analysis instead of individual or aggregations of $\mathrm{H}$ II regions. The latter is required to minimize the effect of contamination due to diffuse gas. If H II regions cannot be segregated, it is not guaranteed that the dominant ionized emission is due to photoionization from young massive stars and therefore, analysis as the abundance derivations are not reliable. This is probably the case for the $Z 2$ case where, due to the low number of pixels, it is difficult to identify
Table 3. Number of H II regions detected for each galaxy.

\begin{tabular}{lccccc}
\hline \hline Galaxy & \multicolumn{6}{c}{ Number of H II regions at $z$} & Z0/Z1 & Z0/Z2 \\
& Z0 & $Z 1$ & $Z 2$ & & \\
\hline NGC 628 & 286 & 77 & 14 & 3.7 & 20.4 \\
NGC 1058 & 331 & 67 & 7 & 4.9 & 47.2 \\
NGC 1637 & 297 & 95 & 14 & 3.1 & 21.2 \\
NGC 3184 & 169 & 66 & 17 & 2.5 & 9.9 \\
NGC 5474 & 122 & 47 & 10 & 2.5 & 12.2 \\
\hline
\end{tabular}

peaky/isolated structures as required by the HIIEXPLORER. As will be shown later for the abundance determination, although for the $Z 2$ case the conditions are not easily fulfilled, the comparison between the spaxel-spaxel and the HIIEXPLORER analysis indicates that the former has a greater misinterpreting possibility, even considering a $\mathrm{S} / \mathrm{N}$ threshold. As already mentioned in Sánchez et al. (2012b), the spatial sampling and resolution of the data presented in this work is not optimal for the derivation of additive properties of individual H II regions. Therefore these data are not optimal for the study of the $\mathrm{H} \alpha$ luminosity function or the characteristic optical extension of H II regions, to mention just a couple of quantities. Although in every analysis of this kind one has to bear in mind that the measurements could correspond to an $\mathrm{H}$ II region complex.

When we reach the higher redshift regime the situation gets more complicated. We are adding together regions belonging to very different galactic components, considering that we are not 
D. Mast et al.: The effects of spatial resolution on integral field spectrograph surveys at different redshifts

Table 4. Distribution of number of regions corresponding to different regimes.

\begin{tabular}{lccc}
\hline \hline Galaxy & Z0 in Z1 & Z0 in Z2 & Z1 in Z2 \\
\hline NGC 628 & $3 \pm 1$ & $20 \pm 5$ & $4 \pm 1$ \\
NGC 1058 & $3 \pm 2$ & $19 \pm 9$ & $5 \pm 2$ \\
NGC 1637 & $2 \pm 1$ & $14 \pm 5$ & $4 \pm 1$ \\
NGC 3184 & $1 \pm 1$ & $4 \pm 3$ & $2 \pm 1$ \\
NGC 5474 & $2 \pm 1$ & $8 \pm 4$ & $3 \pm 1$ \\
\hline
\end{tabular}

distinguishing disc, arms, and bulge, but, in any case, it will depend on the morphology of the studied galaxy.

To explore further the H II region limits mentioned above, we identified all the regions from the higher resolution datacube that are detected as an individual region at lower resolution. In this way we can analyse the real source (or sources) of a particular measurement, and study how it is affected by the spatial binning. Figure 6 shows an example for NGC 628. The upper panel of this figure displays a histogram of the number of $Z 0$ regions inside a $Z 1$ one. This distribution indicates that, on average, each $Z 1$ detected $\mathrm{H}$ II region is built of 3 smaller $Z 0$ regions. For the higher redshift case, each individual region is, in reality, an agglomerate of around 15 to $25 \mathrm{Z0}$ regions, while it is built by the contribution of $\sim 4 Z 1$ regions. Table 4 summarises the distribution of the number of regions in terms of median and standard deviation for all the sample.

\subsubsection{Diagnostic diagrams}

We can use the $[\mathrm{O}$ III $] \lambda 5007 / \mathrm{H} \beta$ ratio, together with $[\mathrm{N}$ II $] \lambda 6583 /$ $\mathrm{H} \alpha$, to construct the classical BPT diagram for all the galaxies, as shown in Fig. 7. These plots allow us to see how the spatial information loss is affecting our understanding and characterisation of the ionisation sources at higher redshift. It is clear that when the idea is to separate, for example, AGN activity from star-formation, one has to consider the degree of contamination due to the area subtended by the spectroscopic aperture. In the present study, where we are dealing with $\mathrm{H}$ II regions, we have the opportunity to map the way that this resolution degradation alters the position of each individual region on the BPT diagram. We have also plotted the Kewley et al. (2001) demarcation curve, often invoked to distinguish between star-forming regions (Fig. 7, below the red line), and other sources of ionisation, such as AGN/shocks/post-AGB stars (above the line) and the Kauffmann et al. (2003) one to indicate the so-called composite zone. In the plots, empty circles correspond to the Z0 case, while full blue circles and orange squares correspond to $Z 1$ and $Z 2$ respectively. We also plot symbol size proportional to the galactocentric distance, i.e. the larger the symbol, the farther from the galaxy centre.

This distribution of regions in the BTP diagram is expected since star-formation is the dominant ionising source in all the selected galaxies. As described in Kewley \& Ellison (2008) the objects lying in the composite region do not require an additional ionising source per se, in the case of a strong nitrogen enrichment. Other ionisation sources, like shocks, AGB star and AGN photoionization, mixed with pure star-formation, can also populate this region. Thus, the intermediate region only indicates that their nature is uncertain, not that the ionising source is definitely different than star-formation. However, for the particular scope of this article, we have preferred a conservative approach and do not consider them in any further discussion.
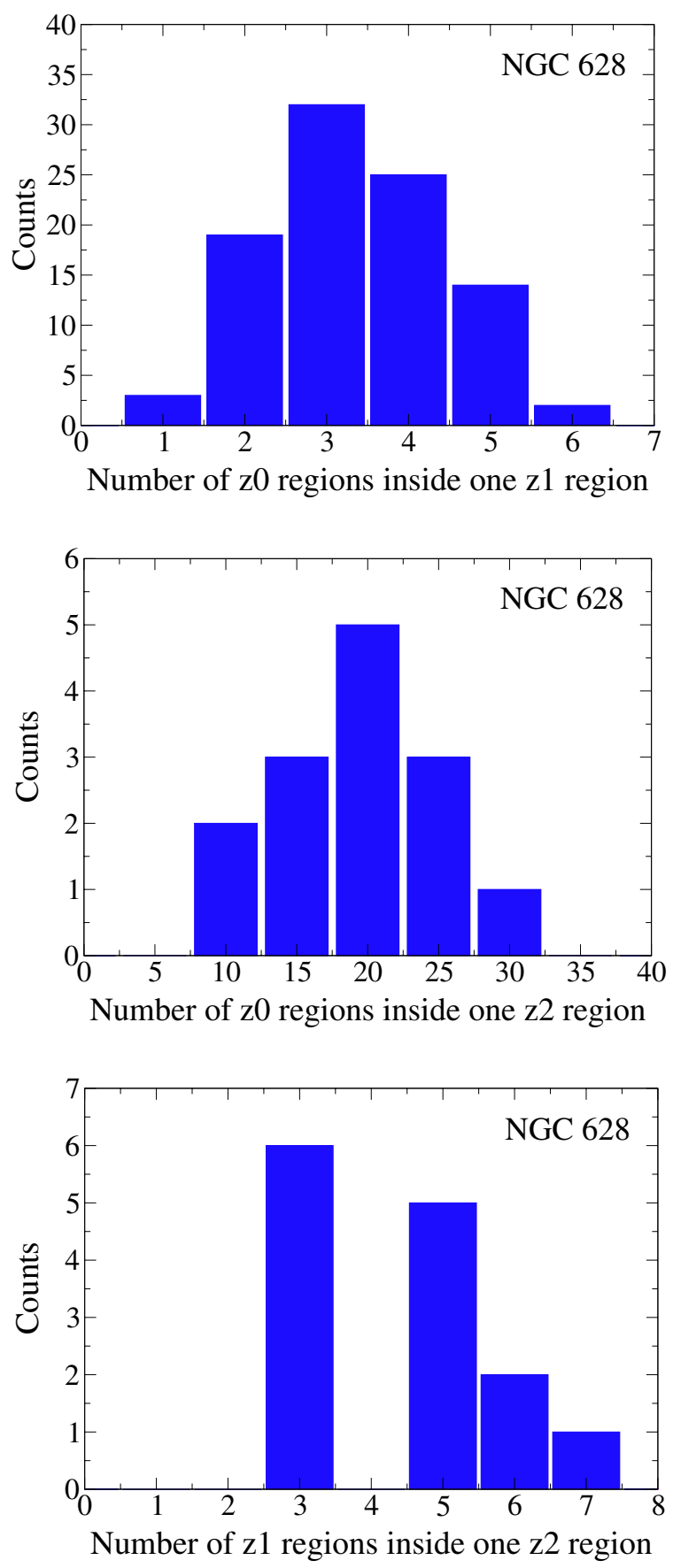

Fig. 6. Correspondence between the numbers of regions in each regime for NGC 628, i.e. the number of regions lying inside an individual region of each other redshift case. Upper panel: correspondence between $Z 0$ and Z1. Middle panel: correspondence between $Z 0$ and $Z 2$. Lower panel: correspondence between $Z 1$ and $Z 2$.

In the $Z 1$ case of NGC 628 (blue circles), the effect of spatial resolution degradations is a collapse of the regions into the most populated zone of the Z0 plot (empty circles), and then again, for the $Z 2$ regime, all regions are more concentrated in the "core" of the lower redshift case. In the $Z 2$ case we do not see composite regions, with all lying in the star formation zone. The same behaviour is displayed in the BPT diagrams of the other galaxies, except for NGC 1637 with several Z2 points in the composite area. The BPT diagram of NGC 1058 displays the strongest collapse effect. Figure 8 shows the behaviour of one individual $Z 2$ region of NGC 628 and its constituents from 

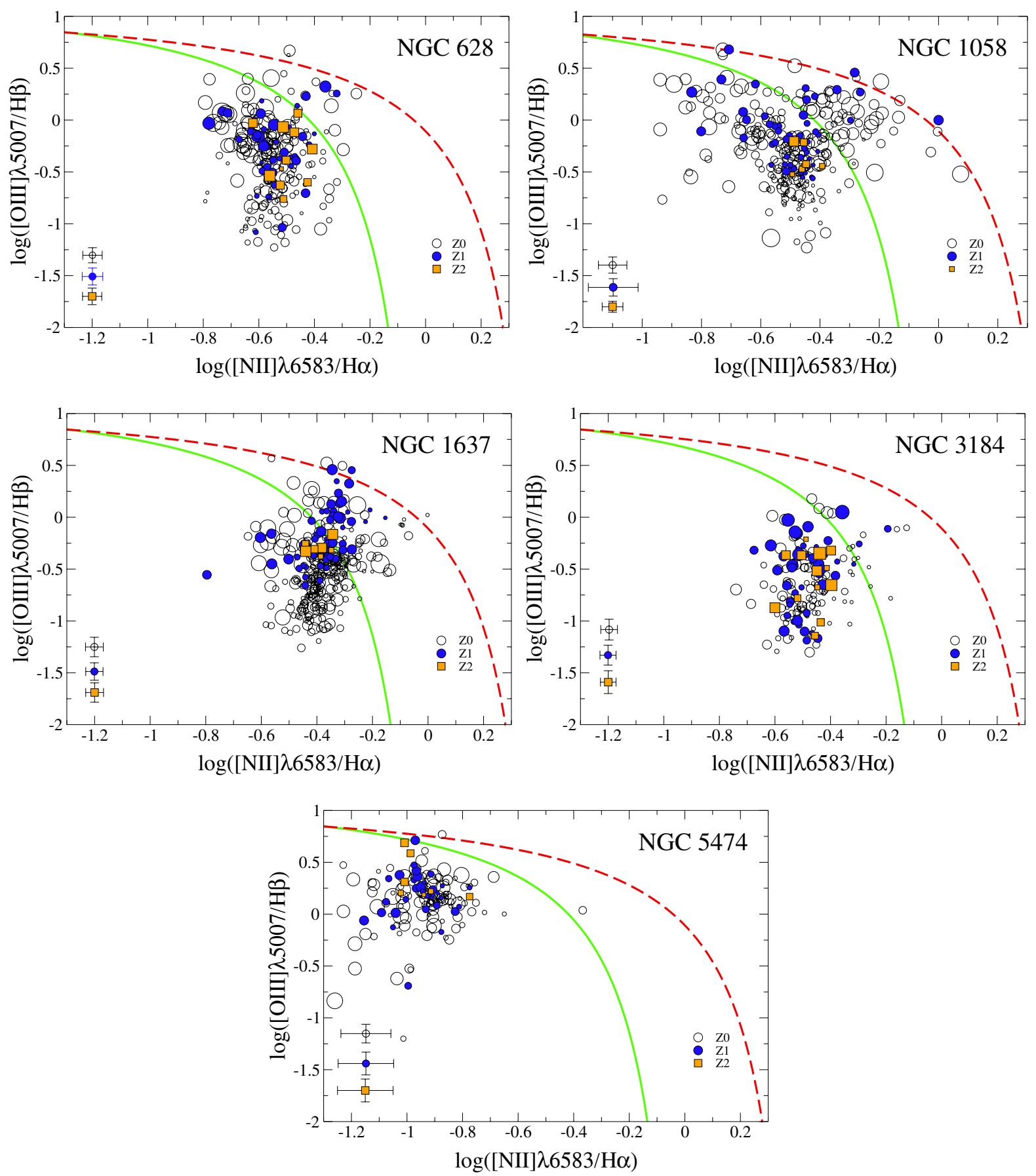

Fig. 7. Classical BPT emission-line diagnostic diagrams (Baldwin et al. 1981; Veilleux \& Osterbrock 1987) with the demarcation curves of Kewley et al. (2001) (upper curve, red) and Kauffmann et al. (2003) (lower curve, green) for each galaxy. Empty circles correspond to Z0, blue circles to $Z 1$, and orange squares to $Z 2$. Symbol sizes are proportional to galactocentric distance, i.e. the larger symbols are, the farther from the centre.

the other redshift regimes. The underlying reason of the shrinking in the occupied parameter space with increasing $Z$ could be because the ratios are luminosity-weighted. If two regions have $R 1=a 1 / b 1$ and $R 2=a 2 / b 2$, then the combined ratio $R=(a 1+a 2) / b=R 1 \times(b 1 / b)+R 2 \times(b 2 / b)$, where $b=b 1+b 2$. In the case where $b 1 \gg b 2(b 1 \ll b 2)$, then $R \sim R 1(R 2)$. When $b 1 \sim b 2$, then $R \sim 0.5 \times(R 1+R 2) . R$ is always between $R 1$ and $R 2$. Therefore, summing multiple H II regions and measuring line ratios mean taking a luminosity-weighted mean of the ratios.

In all the $Z 1$ simulated galaxies the approximate shape of the two-branches structure, visible in the original $Z 0$ galaxies is acceptably discerned. Thus, we suggest that $Z 1$ can recover the global trend of $\mathrm{H}$ II regions in the BPT diagram while Z2 only reflects the denser part of the distribution in the lower redshift case.

\subsubsection{Line ratios and abundances}

For the derivation of the oxygen abundance we adopted the $\mathrm{O} 3 \mathrm{~N} 2$ indicator using the calibration provided by Pettini $\&$ Pagel (2004), defined by the relation $12+\log (\mathrm{OH})=$ 8.73-0.32 $\log _{10}\left(\frac{[\mathrm{O} I I I] \lambda 5007 / \mathrm{H} \beta}{[\mathrm{N} I] / 26583 / \mathrm{H} \alpha}\right)$. This calibration has the advantage of being relatively easy to measure (based on strong 
D. Mast et al.: The effects of spatial resolution on integral field spectrograph surveys at different redshifts

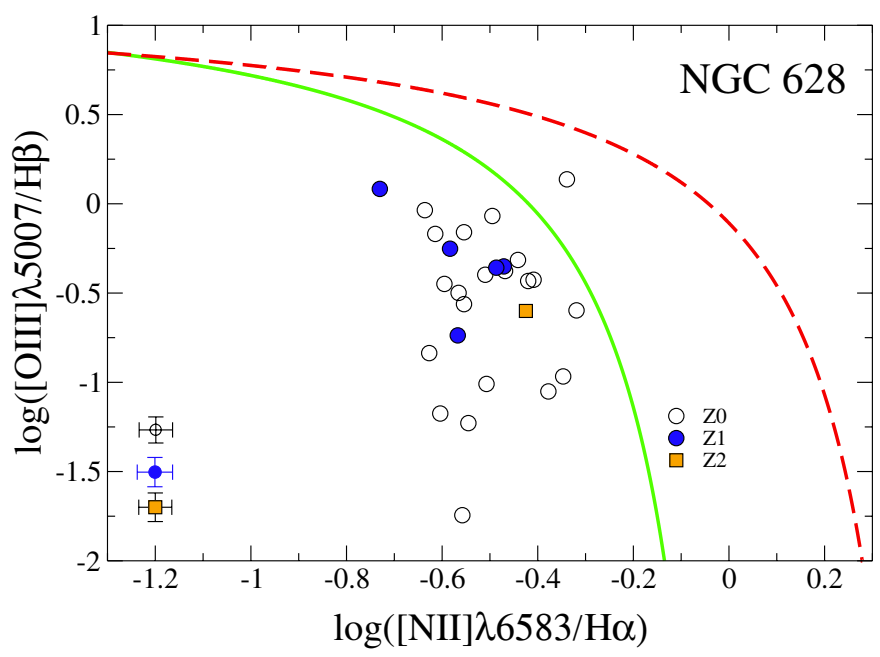

Fig. 8. Same as Fig. 7 but for one $Z 2$ region and its corresponding $Z 0$ and $Z 1$ region content. Empty circles correspond to $Z 0$, blue circles to $Z 1$, and orange squares to $Z 2$.
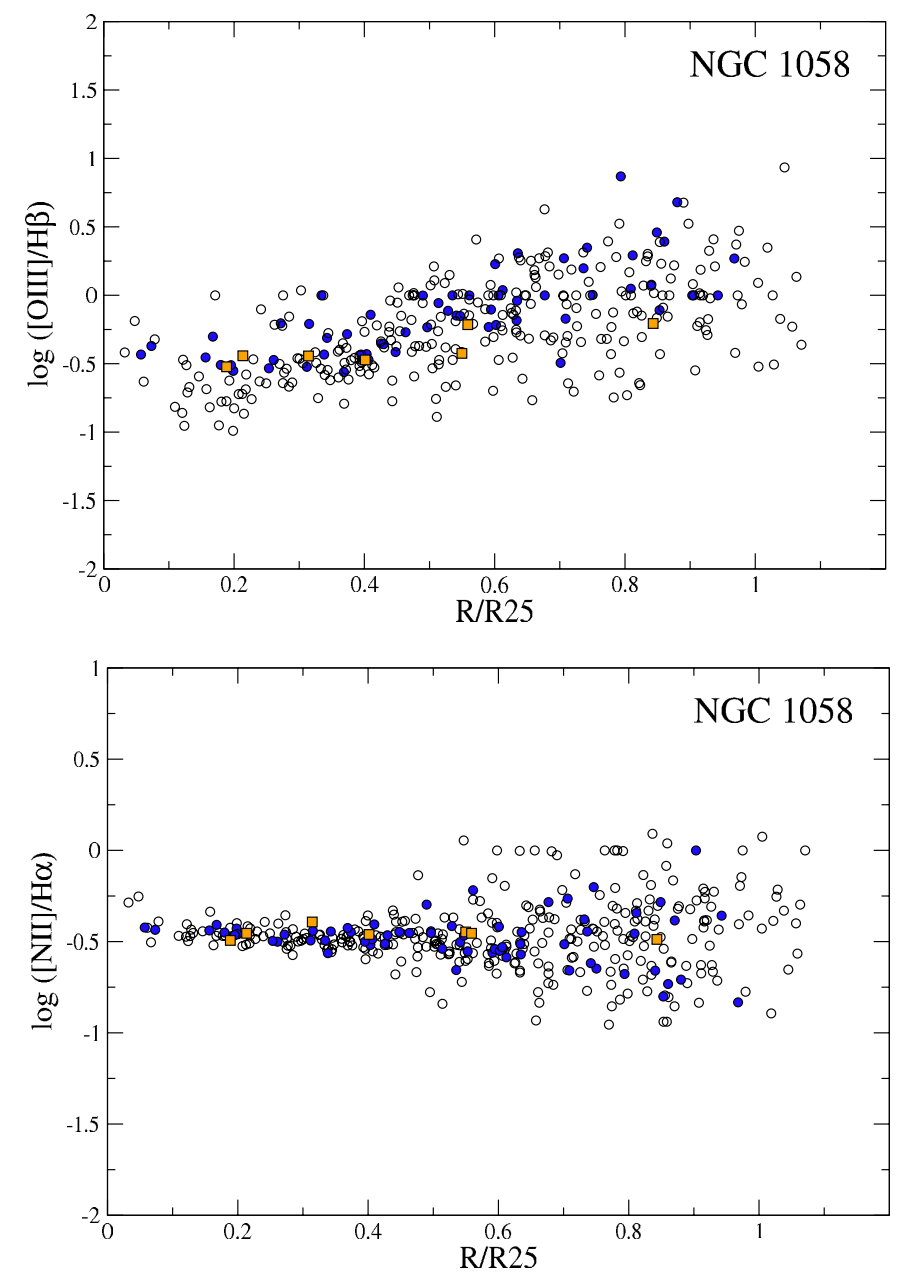

Fig. 9. Top: radial distribution of $[\mathrm{O} \mathrm{III}] / \mathrm{H} \beta$ for NGC 1058. Bottom: radial distribution of the $[\mathrm{N} \mathrm{II}] / \mathrm{H} \alpha$ ratio for the same galaxy. Empty circles correspond to $Z 0$, blue circles to $Z 1$, and orange squares to $Z 2$.

emission lines), is basically not affected by the effects of extinction (the involved line-ratios are very close in wavelength), and implies a simple linear conversion, reason for which this indicator is very popular for high redshift studies ${ }^{3}$ Fig. 9 shows the radial distributions of the ratios $[\mathrm{N} \mathrm{II}] / \mathrm{H} \alpha$ and $[\mathrm{O} \mathrm{III}] / \mathrm{H} \beta$. We can see that the $[\mathrm{O}$ III $] / \mathrm{H} \beta$ radial gradient becomes flatter with redshift. This will have a clear effect on the abundance determination. As an example, Fig. 10 shows the radial abundances for regions in two of the galaxies (NGC 1058 and NGC 1637) with fitted gradients for comparison purposes, corresponding the empty circles to $Z 0$, the blue circles to $Z 1$, and the orange squares to $Z 2$ (see Fig. A.1 for the remaining objects).

We note that the oxygen abundance is not an additive but a relative property, and it exhibits a ubiquitous radial gradient (e.g. Sánchez et al. 2012b). Therefore, neither the average nor a value at a fixed aperture are characteristic of the full distribution (e.g. Tremonti et al. 2004; Rosales-Ortega et al. 2012). Even more, it is still not clear that the average of the values derived at different radii is representative of a single value derived using an aperture that encircles all the previous ones.

In all the galaxies we see a radial abundance gradient decreasing outwards, with NGC 628 and NGC 1058 being the more evident cases. This effect is expected as late-type galaxies are known to have a composition gradient related to their star formation history (SFH). The capability of any survey of being able to measure this gradient is essential if one of its goals is to characterise the properties of galactic discs.

All galaxies show a nearly flat distribution in the innermost parts, until $r \sim 0.2 R_{25}$. From this point, the abundance measurements start to exhibit higher values, reaching maxima at $r \sim 0.4 R_{25}$ in 3 of the cases $\left(r \sim 0.2 R_{25}\right.$ for NGC 1058). After this, the slope of the radial abundance distribution changes its sign, and decreases with increasing galactocentric radius. In all cases, $Z 1$ reproduces notably the $Z 0$ trend with slightly lower abundances. The same radial gradients are visible, with the only difference being the absence of the peak at $r \sim 0.2 R_{25}$ (in the case of NGC 1058) and $r \sim 0.4 R_{25}$ (for NGC 628). For all the galaxies, $Z 2$ fails to reproduce the slope behaviour, as anticipated with the $[\mathrm{O} \mathrm{III}] / \mathrm{H} \beta$ line ratio, showing a nearly flatter distribution (on average) like in NGC 1058 and NGC 1637 (see Fig. 10).

Figure 10 shows that for the higher redshift simulation the abundance flattens. To search for possible sources of this behaviour on the radial abundance distribution for Z2, we performed several tests. We used the H II regions catalogue of Sánchez et al. (2012b) to simulate a spatial binning adding together all the HII regions inside different apertures. The observed effect was a simple average of the H II region properties as expected, discarding the sum of $\mathrm{HII}$ regions as source of the flattening. This can be also confirmed in Fig. 11 that shows the radial abundance gradient of NGC 1058 for the three redshift regimes. Colour coded is each different $Z 2$ region with its corresponding $Z 0$ and $Z 1$ components (same colour). Small circles correspond to $Z 0$, medium sized to $Z 1$ and the bigger ones to $Z 2$. Plus signs represent the abundance determined summing the flux of all the $Z 0$ regions inside the corresponding $Z 2$ region, while

\footnotetext{
3 This indicator has been recently re-calibrated for the high-metallicity regime by Marino et al. (2013). Although there is a known discrepancy in deriving metallicity with different calibrators (Kewley \& Ellison 2008), Sánchez et al. (2012b) showed that there is a correlation between the different determinations, the discrepancies present at high redshift being, to a large degree, a consequence of resolution effects. In any case, although the specific results obtained here are applicable to the O3N2 calibrator in particular, the use of one or the other calibration does not modify qualitatively the results of the proposed analysis. Other methods will suffer from different systematic effects (e.g. absolute scale differences), which analysis are beyond the scope of this paper.
} 

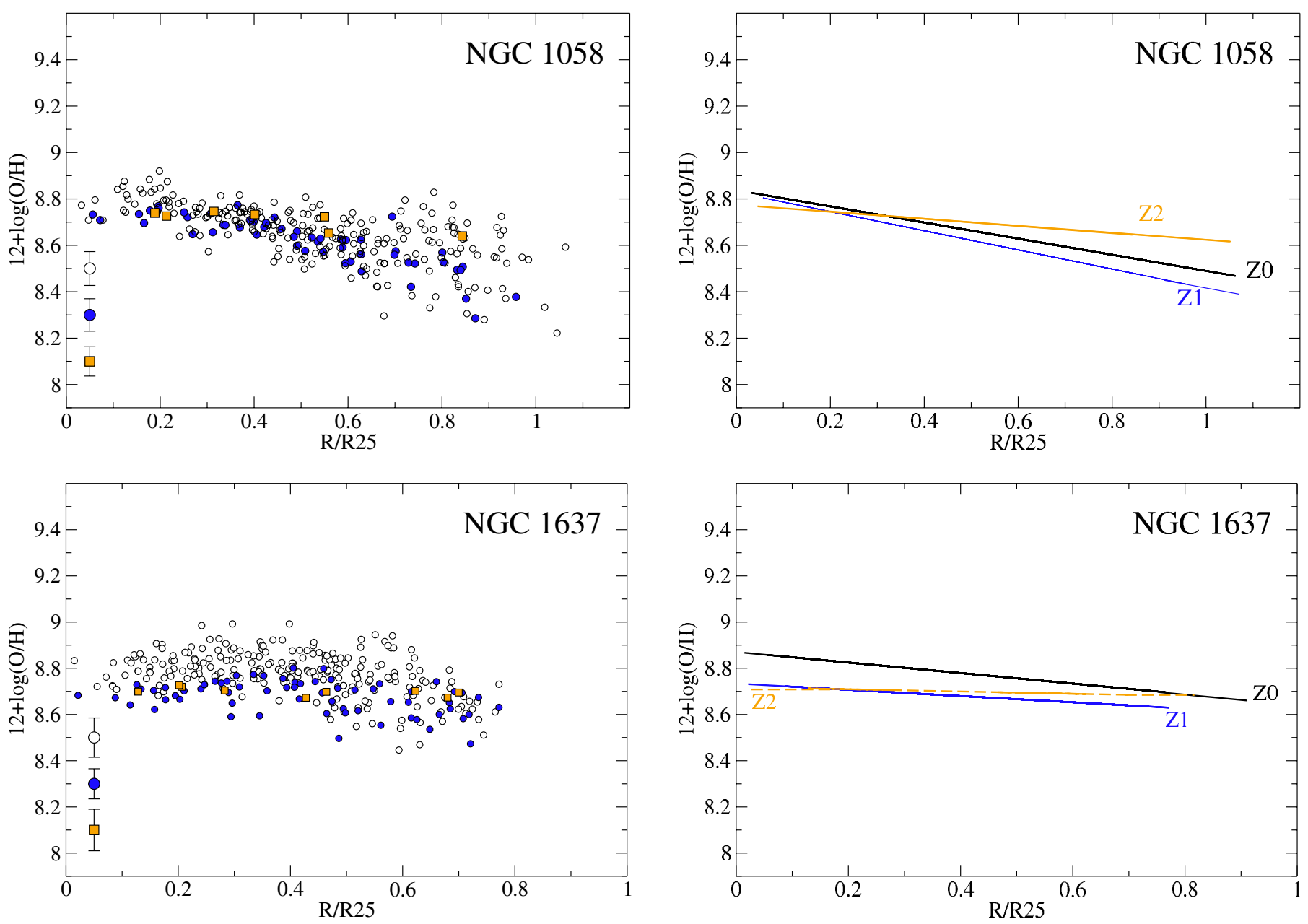

Fig. 10. Radial abundance gradients derived using the relation O3N2 from Pettini \& Pagel (2004) for two of the galaxies. In each left panel the three redshift regimes are over-plotted, the empty circles correspond to $Z 0$, the blue circles to $Z 1$, and the orange squares to $Z 2$. The three points in the bottom left corner show the average error bars. In the right panels we can see a linear regression fitting to each regime.

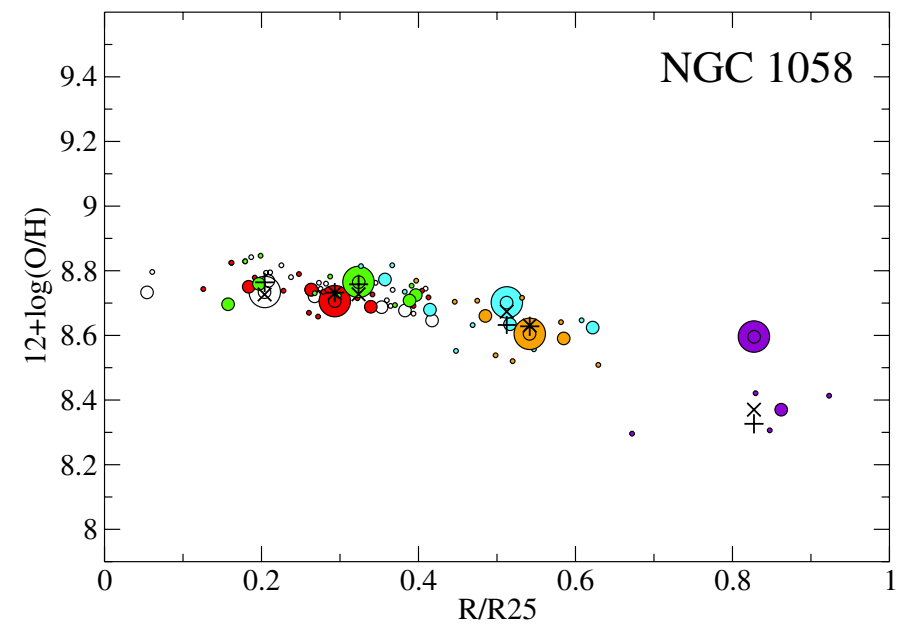

Fig. 11. Radial abundance gradient of NGC 1058 for the three redshift regimes. Colour coded is each different $Z 2$ region with its corresponding $Z 0$ and $Z 1$ components (same colour). Small circles correspond to $Z 0$, medium sized to $Z 1$ and the bigger ones to $Z 2$. Plus signs represent the abundance determined summing the flux of all the $Z 0$ regions inside the corresponding $Z 2$ region, while crosses are the determination from the total flux of $Z 1$ regions (also the ones inside the $Z 2$ region). crosses are the determination from the total flux of the $Z 1$ regions inside that $Z 2$ region.

It is well known that the derivation of Balmer emission line fluxes are affected by the accuracy of the subtraction of the underlying stellar population. In particular, $\mathrm{H} \beta$ is strongly affected. Along the pilot studies for the CALIFA survey (Mármol-Queraltó et al. 2011), studied the capability of recovering this emission line at different equivalent widths, underlying stellar populations, and spectral resolutions. We noticed that at low equivalent widths $\mathrm{H} \beta$ is overestimated for old stellar populations and underestimated for young stellar populations. This effect is stronger at lower spectral resolutions. The net effect of the decrease in the spatial sampling produces these three combined effects: (i) it reduces the original equivalent width by increasing more regions dominated by low-intensity diffuse emission, increasing the underlying continuum with respect to the gas emission; (ii) it increases the fraction of old stellar populations in the inner regions, and the fraction of young stellar populations in the outer ones; and (iii) it decreases the effective spectral resolution by the beam effect, i.e. the co-adding of regions with different kinematic properties. All together it produces an increase in $[\mathrm{O} \mathrm{III}] / \mathrm{H} \beta$ in the inner regions and its decrease in the outer ones. For galaxies with a positive gradient in $[\mathrm{N} \mathrm{II}] / \mathrm{H} \alpha$ it produces a flattening of the radial abundance. 
D. Mast et al.: The effects of spatial resolution on integral field spectrograph surveys at different redshifts

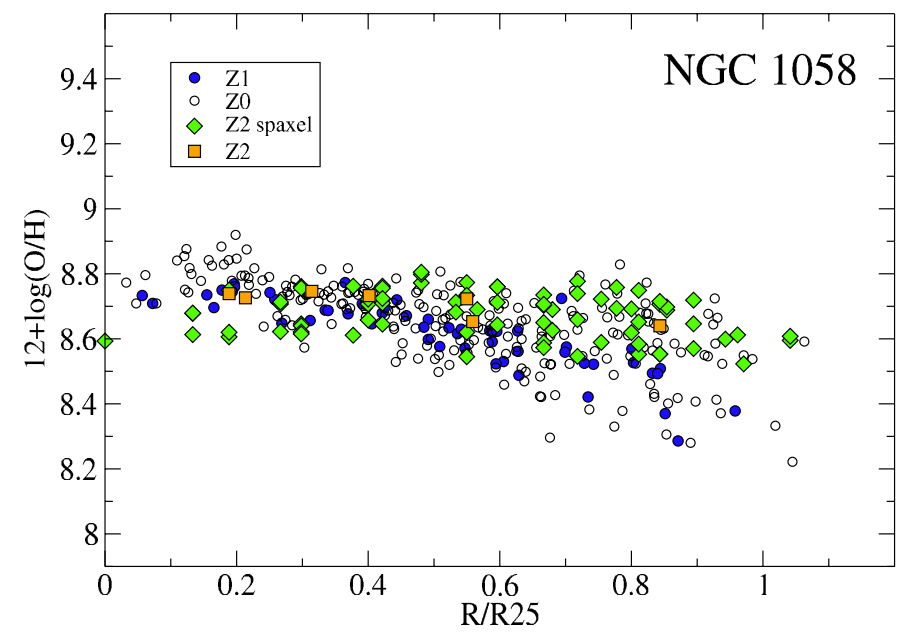

Fig. 12. Comparison between the same analysis as Fig. 10 and a spaxelspaxel determination above a S/N threshold (green) for NGC 1058.

This effect is also present in other abundance indicators, like $R 23$, and less affected by others like $[\mathrm{N} \mathrm{II}] / \mathrm{H} \alpha$. Evidently, the effect of the spatial resolution degradation on the $Z 2$ regime inhibits any possibility of measuring convincing radial abundance gradients at that redshift.

Yuan et al. (2013) find similar results studying the $[\mathrm{N} \mathrm{II}] / \mathrm{H} \alpha$ ratio. They show that seeing-limited observations produce significantly flatter gradients than higher angular resolution observations. They find a critical angular resolution FWHM range $\left(<0.02^{\prime \prime}\right)$, which depends on the intrinsic gradient of the galaxy, beyond which the measured metallicity is significantly more flattened than the intrinsic metallicity.

Another effect that may be playing an important role on the radial abundance gradients and the BPT diagrams, is that when we go to higher redshift, the coarse resolution causes us to add more diffuse gaseous component to the H II aggregates, with potentially different dominating ionising sources than in the H II complexes. The difference between the plus sign, the cross and the bigger circles of Fig. 11 would account for the different amount of diffuse medium considered on each region. The effect of this contamination is very clear if we study cases where we can resolve the diffuse medium at tens-of-parsec scale like in M33 or NGC 5253 (e.g. Voges \& Walterbos 2006; Relaño et al. 2010; Monreal-Ibero et al. 2010a, 2011). In all the mentioned cases, as we move away from the $\mathrm{H}$ II region, with the maximum local $\mathrm{H} \alpha$ flux, as this flux decreases, the ratio [N II] $/ \mathrm{H} \alpha$ increases while $[\mathrm{O}$ III $] / \mathrm{H} \beta$ decreases. This will cause the abundance determination based on the $\mathrm{O} 3 \mathrm{~N} 2$ method to increase. If in a considered galaxy the H II region spatial density is low, the contribution of the diffuse component to the line ratios could become very important and even the dominant source of line fluxes, i.e. as could be the case in the outer points of the radial abundance distribution plotted in Fig. 10. In any case, an estimation of the contribution of the diffuse component is not trivial and depends on the different galaxy components considered (arm, inter-arm, bulge, nucleus, etc.).

Figure 12 shows the comparison between the same analysis as Fig. 10 and an spaxel-spaxel determination above a $\mathrm{S} / \mathrm{N}$ threshold. The low abundance inner pixels may alter the gradient determination. Although a deep analysis of the merging H II complexes and the contribution from diffuse ionized gas (DIG) is beyond the scope of this paper, it is worth noting that they are key points in this kind of studies, and any interpretation should consider them. Although the DIG contribution to the integrated flux in some lines has been quantified in large values (e.g. about $30 \%-50 \%$ of ionized gas, as traced by $\mathrm{H} \alpha$, in galaxies is found outside of $\mathrm{H}$ II regions, Thilker et al. 2002; Oey et al. 2007; Haffner et al. 2009; Monreal-Ibero et al. 2010b; Alonso-Herrero et al. 2010), its final contribution to dust corrected luminosity is much lower (e.g. 5\%-30\%, Crocker et al. 2013; Sánchez et al. 2013). The line ratio of the DIG may differ from that of the star-forming regions, being in general located in the LINER-like region of the BTP diagram (Singh et al. 2013; Papaderos et al. 2013), reflecting ionizing conditions that depend on the morphology of the galaxies (e.g. post-AGBs for most of the earlier-type galaxies, Papaderos et al. 2013). Therefore, the inclusion of larger fraction of DIG emission as the resolution is degraded have a clear effect in the line ratios, although it is the not the only effect to take into account.

When the spatial resolution is good enough to resolve the more luminous $\mathrm{H}$ II regions, it is still possible to use techniques of crowded field spectroscopy (Fabrika et al. 2005; Blanc et al. 2009; Kamann et al. 2013). These techniques are extensions to IFS of the well-known PSF-photometric techniques (DAOPhot), and they rely on the basis that the considered regions are basically unresolved at the spatial resolution of the data. Therefore, it is possible to perform a multi-Gaussian 2D fitting, wavelength by wavelength, to model each of the individual HII-regions, and a $2 \mathrm{D}$ polinomial function to take into account the continuum. This procedure will effectively decouple the diffuse from the resolved emission. However, for doing so it is required to distinguish between both components, and, as we argue along this article, this is not feasible with the spatial resolution of the $Z 2$ data. We have still not implemented this procedure in HIIexplorer, but we will have to add that capability to perform more detailed analysis in the future.

It is clear that the effects in the line ratios and the corresponding derivation of the abundance gradients are complex, and involve many different components that are difficult to disentangle. However, the final result may produce a change in this gradient induced by the degradation of the resolution.

With all the considerations made above, the parameters studied for the gaseous phase show that, contrary to the naive picture, the effect of the resolution degradation is not as intuitive as it would be if we were considering simple additive quantities. Some effects detected in abundance gradients or BPT diagrams at high redshift could be due to spatial information loss.

\subsection{Stellar component}

As we explained in Sect. 5, we used FIT3D for separating the gaseous spectra from the underlying stellar population. In the previous sections we showed the analysis for the gaseous phase, and in what follows we are going to deal with the stellar component.

Spectral indices are widely used for the characterisation of stellar population in galaxies (Trager et al. 2000; Gallazzi et al. 2005). It is beyond the scope of this paper to analyse which method is most suitable for the detail study of stellar population. In the present study we chose the $\mathrm{D}_{4000}$ index to analyse how their derived properties vary in the different redshift regimes. $\mathrm{D}_{4000}$ is a good tracer of stellar age, besides being modelindependent. Errors were determined running a Montecarlo simulation during the fitting procedure. 

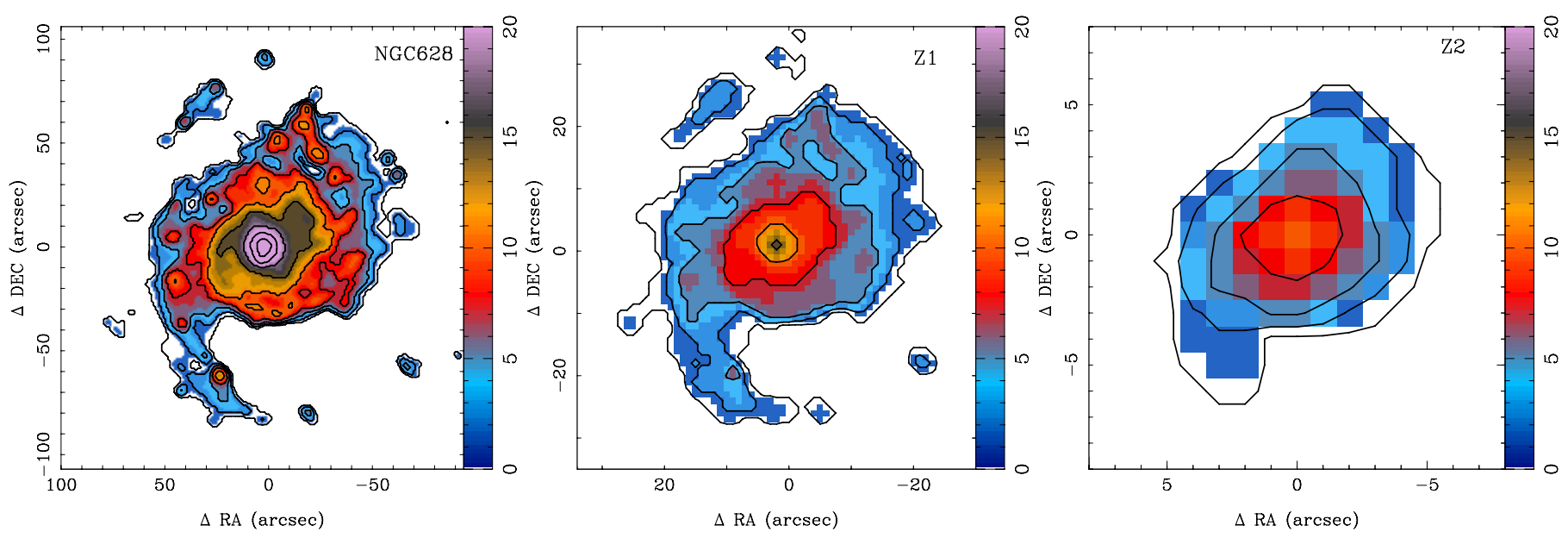

Fig. 13. Isophotal fit performed to $V$-band images extracted from the NGC 628 datacubes. Flux scale is in arbitrary units. For clarity, only some isophotes (black lines) are depicted on the plots. This fitting is later used as segmentation maps for the spectral extraction, i.e the extraction will be performed adding all the spectra between two isophotes separated by a given flux step. North is up, east is to the left. Left: Z0, centre: Z1, right: Z2.

The chosen approach was to generate segmentation maps doing an isophotal fitting to the $V$-band images extracted from the datacubes. The basic idea of any binning for the analysis of data is to increase the $\mathrm{S} / \mathrm{N}$ as much as possible while minimising the degradation of the spatial information. The most widely adopted binning procedure for the analysis of IFS data is the one described by Cappellari \& Copin (2003). This technique performs a binning of the data intended to increase the $\mathrm{S} / \mathrm{N}$ of the low-surface brightness areas, considering spatial vicinity. However, it does not take into account the morphology (relative intensity) between adjacent pixels, which is understandable since it was mostly adopted for the analysis of early-type galaxies (SAURON/ATLAS3D data). For the analysis of galaxies rich in structure (like the ones included in the current study), we consider that a more representative binning should take into account the relative intensity of adjacent pixels.

A similar effect is shown in the standard procedures to derive isophotal distribution of properties (e.g. surface-brightness profiles as the one described in Sect. 5.1). They well describe the azimuthal distribution of properties for early type galaxies, but they do not work as well for late type galaxies.

Therefore, we propose a more simple method to derive isophotal information similar to the one introduced by Papaderos et al. (2002). Instead of assuming a certain shape for the isophote at a certain radii, we just slice the intensity maps on bins of equal intensity, within a certain percentage. Starting from the peak emission of a galaxy, and selecting the range of adjacent pixels to be aggregated within a selected percentage of flux with respect to the peak emission, and iterating until reaching a certain surface brightness, it is possible to bin the data in isophotal areas without any assumption on their shape. Once segregated the galaxy in isophotal areas, it is possible to extract the corresponding coadded spectra, and analyse them. For a given isophotal bin, the distance considered is the distance from the galaxy centre to the average distance to each isophote. This technique provides azimuthal distributions of properties that describe better the actual shape of the galaxies.

If in addition to this simple criteria, a maximum distance between adjacent pixels is considered, then we will end-up with a $2 \mathrm{D}$ binning technique that preserves the actual shape of the galaxies. The details of the technique and its comparison with other methods will be described elsewhere.
In Fig. 13 we can see an example for this segmentation method for NGC 628. As described in detail in Sánchez et al. (2007), FIT3D measures the stellar indices from the extracted stellar spectra, normalised to the standard Lick/IDS resolution.

Figure 14 displays the radial distribution of the $\mathrm{D}_{4000}$ index for all of the sample. Being sensitive to stellar age, this index is higher in the presence of an older stellar population.

For all the galaxies, the $Z 0$ radial behaviour of the $D_{4000}$ index (open circles) is totally reproduced by the $Z 1$ regime (blue circles). Only the innermost regions, when a sharp structure is present, it is slightly smeared out in the $Z 1$ case. The higher redshift regime (orange squares) follows the radial trend when the distribution is smooth, as is the case of NGC 1058. Z2 is unable to reproduce the innermost structures presented in NGC 628, NGC 3184 and NGC 1637.

All these indices are luminosity weighted. Summing two spaxels means performing a luminosity-weighted average of their $\mathrm{D}_{4000}$ break strength, so the observed behaviour is more intuitive than the one for the more complex emission line ratios. Unlike the gaseous phase where we have the information in clumps, the stellar component contains the information in a smooth, less clumpy way. In the cases where the radial $\mathrm{D}_{4000}$ distribution is flatter, the spatial resolution loss has a small effect at higher redshifts. This behaviour allows the higher redshift regime to follow the radial trend without much scatter, although loosing any fine detail present in the distribution.

We conducted similar test with other stellar indices as $\mathrm{H} \delta$ index, i.e. the equivalent width of the Balmer line $\mathrm{H} \delta$, and the $[\mathrm{MgFe}]$ index $\left([\mathrm{MgFe}]=\sqrt{\mathrm{Mg} b\left(0.72 \mathrm{Fe}_{5270}+0.28 \mathrm{Fe}_{5335}\right)}\right) . \mathrm{As}$ a result of our low spectral resolution we are not able to confirm if complex indices based on absorption lines (as $[\mathrm{MgFe}]$ ) are traceable to higher redshift, but we can conclude that simple ones like $\mathrm{H} \delta$ or $\mathrm{D}_{4000}$ are.

\section{Summary and conclusions}

In this paper we have studied how the information loss due to spatial resolution degradation would affect IFS surveys at different redshifts. For this purpose we used a sample of five PINGS galaxies (Z0) and simulated two redshift regimes without taking into account surface brightness dimming or increase 
D. Mast et al.: The effects of spatial resolution on integral field spectrograph surveys at different redshifts
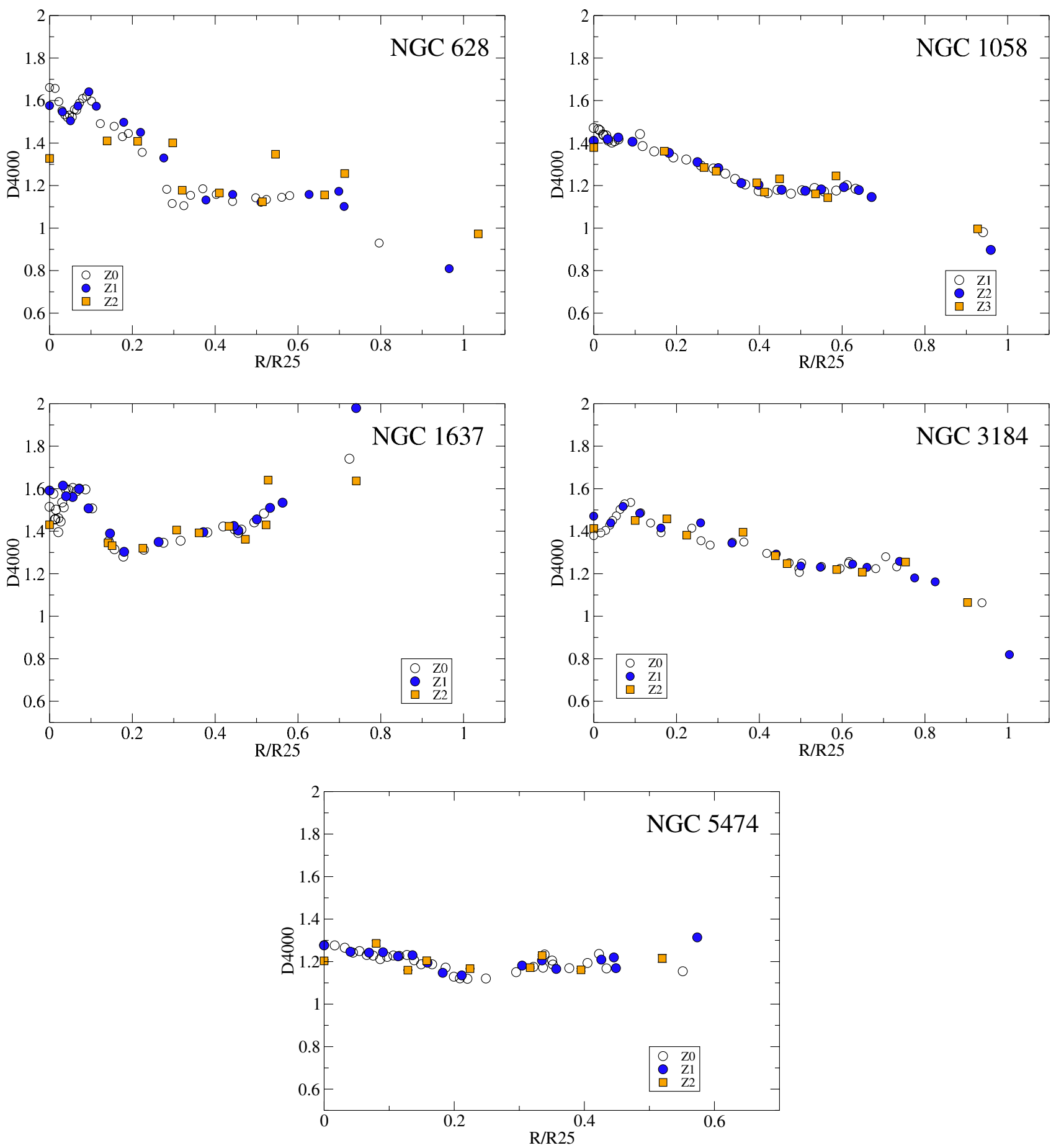

Fig. 14. Radial distribution of the $\mathrm{D}_{4000}$ index defined as the ratio of the flux in the red continuum to that in the blue continuum. Black empty circles correspond to $Z 0$ regime, blue circles to $Z 1$, and orange squares to $Z 2$. The error bars are similar to the symbol sizes.

in noise. One associated with the ongoing CALIFA survey $(Z 1)$ and the other with an hypothetical higher redshift survey $(Z 2)$. We then performed the same analysis to the $Z 0$ galaxies and their simulated versions. We studied the behaviour of the radial abundance (through the O3N2 method), BPT diagrams, and one spectral index $\mathrm{D}_{4000}$, in addition to the $\mathrm{H} \alpha$ emission and the morphology. Our main conclusions regarding each measured quantity, can be summarised as follows:

- Morphology: Z1 is able to reproduce all morphological signatures visible at lower redshift. Despite the detail loss (implying that several individual regions or hot spots are not detected as such), spiral arms, rings and bulge extensions can be traced perfectly. For the $Z 2$ regime the identification of spiral signatures is complicated, and only possible if the structure is a strong morphological feature on a global scale in the galaxy. Several of the higher redshift examples shown in this paper present disturbed morphology that prevents from doing a reliable morphological classification.

- HII regions detection: the implemented method of $\mathrm{H}$ II region detection with HIIEXPLORER, showed that $Z 1$ is detecting nearly $1 / 3$ of the number of original $Z 0$ regions, 
and $Z 2 \sim 1 / 5$ of the number of regions detected at the $Z 1$ redshift (hence, $\sim 1 / 15$ of $Z 0$ ). This illustrates what is the underestimation of the number of $\mathrm{H}$ II regions due the loss of spatial resolution.

- Diagnostic diagrams: the BPT diagrams showed that the effect of spatial resolution degradation on this diagrams is to collapse the measured values into the denser regions of $Z 0$ plots. $Z 1$ is able to reproduce with acceptable accuracy the shape of the $Z 0 \mathrm{BPT}$. For the $Z 2$ situation, only the most populated regions of the lower redshift regime are mapped. Despite the displacement observed, the main ionization mechanism of the observed galaxy at the putative higher redshift is mostly due to thermal ionization from hot massive young stars.

- Radial abundance: The O3N2 method (commonly used at high redshift to derive gas metallicity) was applied to our sample. It showed that the flat inner part of the abundance distribution presented in the $Z 0$ galaxies is observable in the $Z 1$ case and, although with smoothed values, in $Z 2$. $Z 1$ and $Z 2$ are able to reproduce the maximum value displayed around $(0.2-0.4) R_{25}$ in the $Z 0$ galaxies. The gradient slopes in $Z 0$ are slightly smoothed in $Z 1$ but still present. In the higher redshift case global trends are traceable. We note that the degradation and contamination of H II complexes at higher redshift may induce spurious radial trends.

- Spectral indices: for the analysed index $\mathrm{D}_{4000}$, global tendencies are correctly traced on both simulated cases, and $Z 1$ is also capable, at some level, of reproducing fine structure of the $Z 0$ distributions.

As a global conclusion, we showed that the information loss will depend on the level of detail contained in the analysed feature. In this sense, if the studied galaxy has a smooth profile, earlytype, with smooth behaviour in their properties, that behaviour will still be visible at higher redshift. But any sharp structure will be lost. Our analysis allows us to conclude that CALIFA will be able to analyse to an acceptable scale, and with a good level of detail, all desired magnitudes, in its aim of characterising the Local Universe. For the hypothetical higher redshift survey, the perspectives are difficult but promising, since global trends, averaged values, and, in some cases, local structures, are acceptably mapped and correctly interpreted in the considered framework.

It is worth noting that the important figure of merit is the ratio between the spaxel size and the typical scale-length at a certain redshift. As examples of hypothetical $Z 2$ surveys, we can consider SAMI (1.6"/fibre) or MaNGA (3"/fibre). In this regard, the conclusions obtained for the $Z 2$ simulation are also applicable to other redshift ranges depending on the instrument. For example, if we are studying galaxies with $\sim 0.25 \mathrm{kpc} / \mathrm{spaxel}$, we have shown that most of the common observables are perfectly traced, while this is not the case with a scale of $1 \sim \mathrm{kpc} / \mathrm{spaxel}$.

As real examples, we can consider the Gemini Multi-Object Spectrographs (GMOS; Allington-Smith et al. 2002). With $25 \times$ 35 spaxels and a $0.2^{\prime \prime} /$ spaxel scale our results showed that at $z \sim 0.05$ it is still possible to derive radial gradients, whenever the right depth and spatial coverage are granted. The same conclusion can be applied to VIMOS (VIsible MultiObject Spectrograph, Le Fèvre et al. 2003) in the Medium Resolution mode (a $13^{\prime \prime} \times 13^{\prime \prime}$ FoV with $0.33^{\prime \prime} /$ spaxel). But, as we have already shown, at $z \sim 0.4$, for example, none of these instruments is capable of measuring properly any of the observables studied. Another positive example could be SINFONI (Spectrograph for INtegral Field Observations in the Near
Infrared, Eisenhauer et al. 2003) in its $8^{\prime \prime} \times 8^{\prime \prime}$ FoV with a $0.125^{\prime \prime} \times 0.125^{\prime \prime}$ spaxel, or even with the $3^{\prime \prime} \times 3^{\prime \prime}$ mode, provided that the Adaptive Optics Facility is used, $z \sim 0.1$ can be reached with considerable success. In the usual redshift ranges that SINFONI has been used $(z \sim 1)$, we have shown that no convincing metallicity radial gradients can be obtained with the data.

Acknowledgements. We would like to thank the anonymous referee for his/her useful comments that have significantly improved the first submitted version of this paper. Based on observations collected at the Centro Astronómico HispanoAlemán (CAHA) at Calar Alto, operated jointly by the Max-Planck Institut für Astronomie and the Instituto de Astrofísica de Andalucía (CSIC). D.M. and A.M.-I. are supported by the Spanish Research Council within the programme JAE-Doc, Junta para la Ampliación de Estudios, co-funded by the FSE. F.F.R.O. acknowledges financial support from the Mexican National Council for Science and Technology (CONACYT) under the programme Estancias Posdoctorales y Sabáticas al Extranjero para la Consolidación de Grupos de Investigación, 2010-2012. I.M. acknowledges financial support from the Spanish MINECO grant AYA 2010-15169, and from Junta de Andalucía TIC114 and Proyecto de Excelencia P08-TIC-03531; J.F.-B. from the Ramón y Cajal Program, grants AYA2010-21322-C03-02 and AIB-2010-DE-00227 from the Spanish Ministry of Economy and Competitiveness (MINECO), as well as from the FP7 Marie Curie Actions of the European Commission, via the Initial Training Network DAGAL under REA grant agreement na 289313. C.J.W. acknowledges support through the Marie Curie Career Integration Grant 303912. E.M.Q. and J.F.-B. acknowledge support from the Spanish Programa Nacional de Astronomía y Astrofísica under grant AYA2010-21322-C03-02. This work has been partially funded by the Spanish PNAYA, project AYA2010-21887 of the Spanish MINECO. R.A. Marino was also funded by the Spanish Programme of International Campus of Excellence Moncloa (CEI).

\section{Appendix A: Radial abundance determination for the remaining objects}

In Sect. 5.2.3, for presentation purposes, we showed only two objects. In Fig. A.1 the radial abundance gradients for the remaining galaxies are shown.

\section{Appendix B: Annular binning}

One binning scheme widely used in different surveys and metallicity studies is the annular binning extraction, method which is often used to derive metallicity gradients, specially in low S/N high-z data (e.g., Jones et al. 2010; Queyrel et al. 2012; Swinbank et al. 2012; Yuan et al. 2013). Figure B.1 shows the radial abundance gradients for all the sample, using this binning method. Annuli are one pixel wide for all the redshift regimes. The black line overplotted on the graph represents the abundance determination of Sect. 5.2.3 for the Z0 regime using the HIIEXPLORER. As this method wipe out any azimuthal variation and add together the HII region and the diffuse emission (that in general do no have the same ionizing conditions as the starforming-regions) for a given annulus on all the three regimes, the result is not surprising. Radial distributions have low dispersion compared with the ones in Sect. 5.2.3 and small scale variations are smeared out as resolution gets coarse.

As can be seen from the plots, in the cases where the DIG is not dominated by LINER-like emission, the radial distribution for the three regimes are more similar (despite the small variations), indicating that for these cases the use of the annular binning extraction scheme would be more suitable at higher redshift. In any case it will depend on the morphology and distribution of the HII regions on each particular galaxy, information that in general is not available for high-redshift objects. 
D. Mast et al.: The effects of spatial resolution on integral field spectrograph surveys at different redshifts
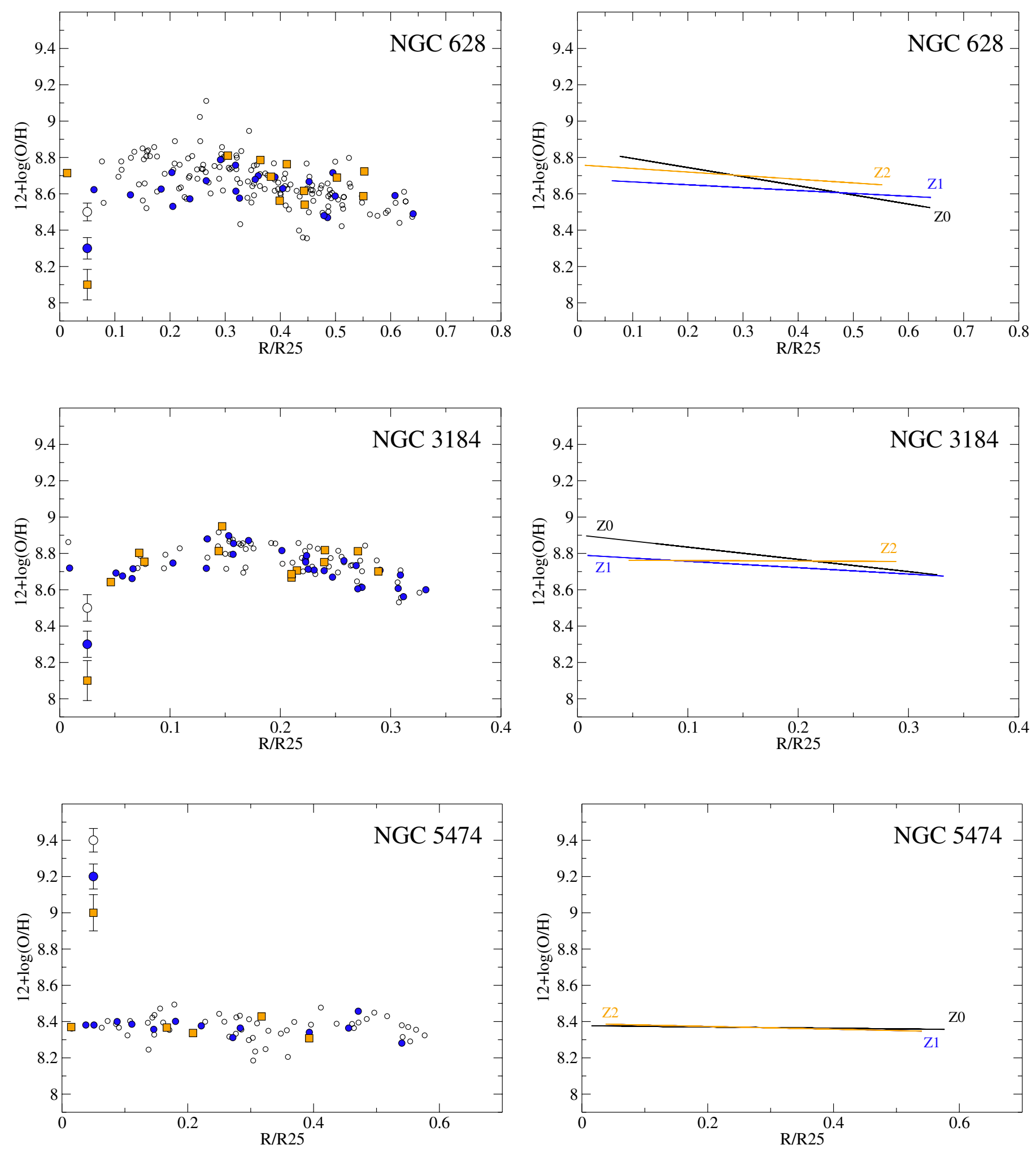

Fig. A.1. Radial abundance gradients derived using the relation O3N2 from Pettini \& Pagel (2004) for the three remaining galaxies. In each left panel the three redshift regimes are over-plotted, the empty circles correspond to $Z 0$, the blue circles to $Z 1$, and the orange squares to $Z 2$. The three points in the lower left corner (upper left for NGC 5474) show the average error bars. In the right panels we can see a linear regression fitting to each regime. 

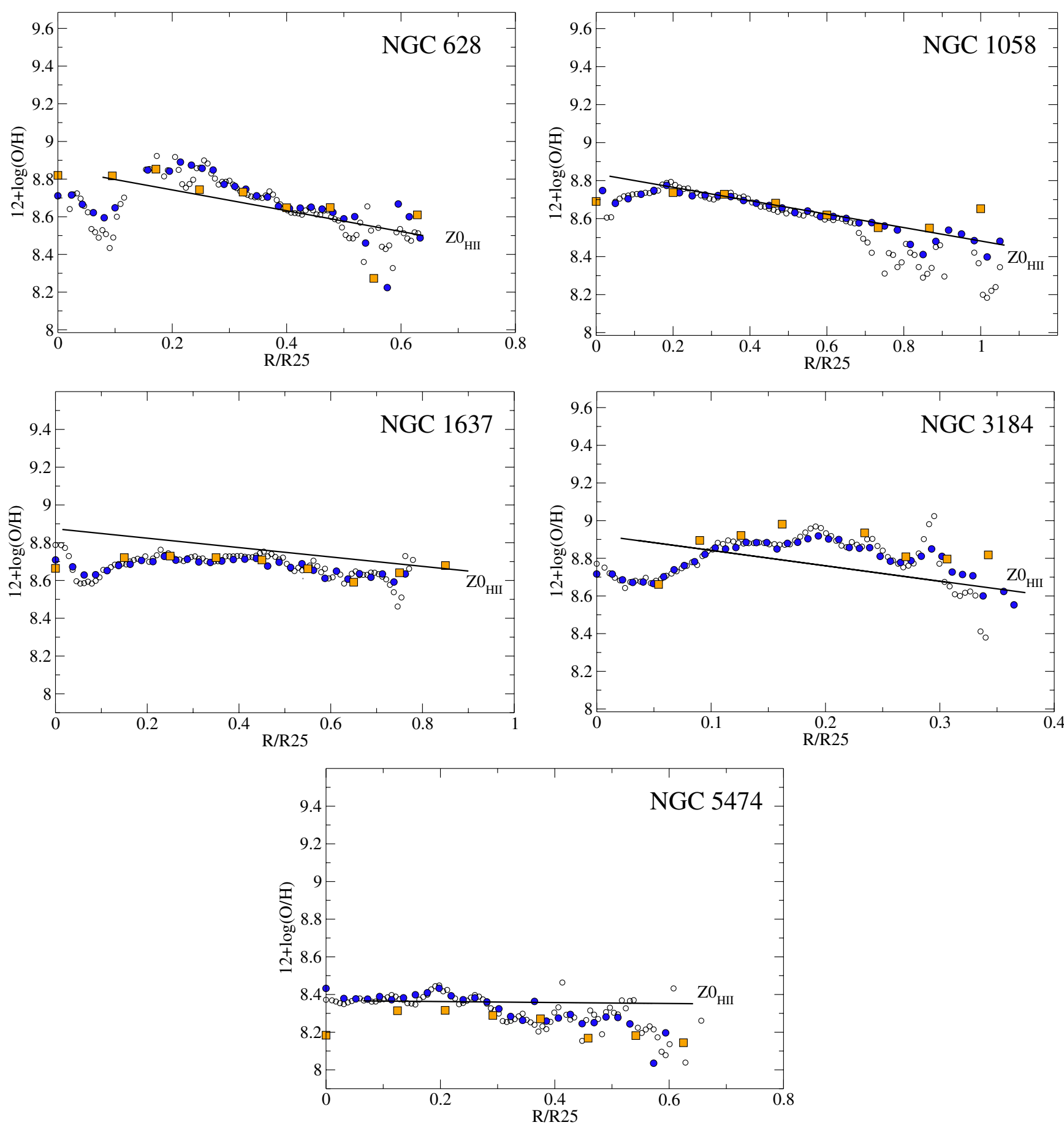

Fig. B.1. Radial abundance gradients for all the sample, using the annular binning scheme for the spectral extraction. On each redshift regime annuli are one pixel wide (on its corresponding scale). In each panel the three redshift regimes are over-plotted, the empty circles correspond to $Z 0$, the blue circles to $Z 1$, and the orange squares to $Z 2$ as usual. The black line indicates the $Z 0$ abundance gradient determined in Sect. 5.2.3.

\section{References}

Allington-Smith, J., Murray, G., Content, R., et al. 2002, PASP, 114, 892 Alonso-Herrero, A., García-Marín, M., Rodríguez Zaurín, J., et al. 2010, A\&A, $522, \mathrm{~A} 7$

Baldwin, J. A., Phillips, M. M., \& Terlevich, R. 1981, PASP, 93, 5

Balogh, M. L., Morris, S. L., Yee, H. K. C., Carlberg, R. G., \& Ellingson, E. 1999, ApJ, 527, 54

Barden, M., Jahnke, K., \& Häumlußler, B. 2008, ApJS, 175, 105

Bennett, C. L., Halpern, M., Hinshaw, G., et al. 2003, ApJS, 148, 1

Bershady, M. A., Verheijen, M. A. W., Swaters, R. A., et al. 2010, ApJ, 716, 198 Blanc, G. A., Heiderman, A., Gebhardt, K., Evans, N. J., II, \& Adams, J. 2009, ApJ, 704, 842
Blanc, G. A., Gebhardt, K., Heiderman, A., et al. 2010, New Horizons in Astronomy: Frank N. Bash Symposium 2009, ASP Conf. Ser., 432, 180

Caon, N., Capaccioli, M., D’Onofrio, M., \& Longo, G. 1994, A\&A, 286, L39

Cappellari, M., \& Copin, Y. 2003, MNRAS, 342, 345

Cappellari, M., Emsellem, E., Krajnović, D., et al. 2011, MNRAS, 413, 813

Cardelli, J. A., Clayton, G. C., \& Mathis, J. S. 1989, ApJ, 345, 245

Casoli, F., Combes, F., Dupraz, C., Gerin, M., \& Boulanger, F. 1986, A\&A, 169, 281

Crocker, A. F., Calzetti, D., Thilker, D. A., et al. 2013, ApJ, 762, 79

Croom, S. M., Lawrence, J. S., Bland-Hawthorn, J., et al. 2012, MNRAS, 421, 872

de Vaucouleurs, G., de Vaucouleurs, A., Corwin, H. G., Jr., et al. 1992, VizieR Online Data Catalog: VII/137B 
de Zeeuw, P. T., Bureau, M., Emsellem, E., et al. 2002, MNRAS, 329, 513 Dutil, Y., \& Roy, J.-R. 1999, ApJ, 516, 62

Eisenhauer, F., Abuter, R., Bickert, K., et al. 2003, Proc. SPIE, 4841, 1548

Fabrika, S., Sholukhova, O., Becker, T., et al. 2005, A\&A, 437, 217

Förster Schreiber, N. M. 2009, in Proc. Unveiling the Mass Extracting and Interpreting Galaxy Masses, id.44

Gallazzi, A., Charlot, S., Brinchmann, J., White, S. D. M., \& Tremonti, C. A. 2005, MNRAS, 362, 41

García-Benito, R., Pérez, E., Díaz, Á. I., Maíz Apellániz, J., \& Cerviño, M. 2011, AJ, 141, 126

Garnett, D. R., \& Shields, G. A. 1987, ApJ, 317, 82

Garnett, D. R., Shields, G. A., Skillman, E. D., Sagan, S. P., \& Dufour, R. J. 1997, ApJ, 489, 63

Genzel, R., Lutz, D., Sturm, E., et al. 1998, ApJ, 498, 579

Giavalisco, M., Livio, M., Bohlin, R. C., Macchetto, F. D., \& Stecher, T. P. 1996, AJ, 112, 369

González Delgado, R. M., \& Pérez, E. 1997, ApJS, 108, 199

Graham, A. W., Driver, S. P., Petrosian, V., et al. 2005, AJ, 130, 1535

Graham, A. W., Merritt, D., Moore, B., Diemand, J., \& Terzić, B. 2006, AJ, 132, 2711

Haffner, L. M., Dettmar, R.-J., Beckman, J. E., et al. 2009, Rev. Mod. Phys., 81, 969

Hendry, M. A., Smartt, S. J., Maund, J. R., et al. 2005, MNRAS, 359, 906

Jones, T., Ellis, R., Jullo, E., \& Richard, J. 2010, ApJ, 725, L176

Kamann, S., Wisotzki, L., \& Roth, M. M. 2013, A\&A, 549, A71

Kauffmann, G., Heckman, T. M., Tremonti, C., et al. 2003, MNRAS, 346, 1055

Kelz, A., \& Roth, M. M. 2006, New Astron. Rev., 50, 355

Kelz, A., Verheijen, M. A. W., Roth, M. M., et al. 2006, PASP, 118, 129

Kennicutt, R. C., Jr. 1998, ARA\&A, 36, 189

Kennicutt, R. C., Jr., \& Garnett, D. R. 1996, ApJ, 456, 504

Kewley, L. J., \& Ellison, S. L. 2008, ApJ, 681, 1183

Kewley, L. J., Dopita, M. A., Sutherland, R. S., Heisler, C. A., \& Trevena, J. 2001, ApJ, 556, 121

Kewley, L. J., Groves, B., Kauffmann, G., \& Heckman, T. 2006, MNRAS, 372, 961

Kormendy, J. 1977, ApJ, 218, 333

Kronberger, T., Kapferer, W., Schindler, S., \& Ziegler, B. L. 2007, A\&A, 473, 761

Law, D. R., Steidel, C. C., \& Erb, D. K. 2006, AJ, 131, 70

Le Fèvre, O., Saisse, M., Mancini, D., et al. 2003, Proc. SPIE, 4841, 1670

López, L. A., Krumholz, M. R., Bolatto, A. D., Prochaska, J. X., \& RamirezRuiz, E. 2011, ApJ, 731, 91

Marino, R. A., Rosales-Ortega, F. F., Sánchez, S. F., et al. 2013, A\&A, 559, A114

Mármol-Queraltó, E., Sánchez, S. F., Marino, R. A., et al. 2011, A\&A, 534, A8

Monreal-Ibero, A., Vílchez, J. M., Walsh, J. R., \& Muñoz-Tuñón, C. 2010a, A\&A, 517, A27

Monreal-Ibero, A., Arribas, S., Colina, L., et al. 2010b, A\&A, 517, A28

Monreal-Ibero, A., Relaño, M., Kehrig, C., et al. 2011, MNRAS, 413, 2242

Moustakas, J., Kennicutt, R. C., Jr., Tremonti, C. A., et al. 2010, ApJS, 190, 233

Oey, M. S., Parker, J. S., Mikles, V. J., \& Zhang, X. 2003, AJ, 126, 2317

Oey, M. S., Meurer, G. R., Yelda, S., et al. 2007, ApJ, 661, 801

Papaderos, P., Izotov, Y. I., Thuan, T. X., et al. 2002, A\&A, 393, 461

Papaderos, P., Gomes, J. M., Vílchez, J. M., et al. 2013, A\&A, 555, L1

Pettini, M., \& Pagel, B. E. J. 2004, MNRAS, 348, L59

Pilyugin, L. S., Vílchez, J. M., \& Contini, T. 2004, A\&A, 425, 849

Queyrel, J., Contini, T., Kissler-Patig, M., et al. 2012, A\&A, 539, A93

Ravikumar, C. D., Puech, M., Flores, H., et al. 2007, A\&A, 465, 1099

Relaño, M., Monreal-Ibero, A., Vílchez, J. M., \& Kennicutt, R. C. 2010, MNRAS, 402, 1635

Rodríguez Zaurín, J., Tadhunter, C. N., \& González Delgado, R. M. 2008, MNRAS, 384, 875

Rosales-Ortega, F. F., Kennicutt, R. C., Sánchez, S. F., et al. 2010, MNRAS, 405, 735

Rosales-Ortega, F. F., Díaz, A. I., Kennicutt, R. C., \& Sánchez, S. F. 2011, MNRAS, 415, 2439

Rosales-Ortega, F. F., Sánchez, S. F., Iglesias-Páramo, J., et al. 2012, ApJ, 756, L31

Rosolowsky, E., \& Simon, J. D. 2008, ApJ, 675, 1213

Roth, M. M., Kelz, A., Fechner, T., et al. 2005, PASP, 117, 620

Sánchez, S. F. 2004, Astron. Nachr., 325, 167

Sánchez, S. F. 2006, Astron. Nachr., 327, 850

Sánchez, S. F., Cardiel, N., Verheijen, M. A. W., Pedraz, S., \& Covone, G. 2007, MNRAS, 376, 125

Sánchez, S. F., Rosales-Ortega, F. F., Kennicutt, R. C., et al. 2011, MNRAS, 410, 313

Sánchez, S. F., Kennicutt, R. C., Gil de Paz, A., et al. 2012a, A\&A, 538, A8

Sánchez, S. F., Rosales-Ortega, F. F., Marino, R. A., et al. 2012b, A\&A, 546, A2

Sánchez, S. F., Rosales-Ortega, F. F., Jungwiert, B., et al. 2013, A\&A, 554, A58
Sánchez-Portal, M., Cepa, J., Pintos-Castro, I., et al. 2011, in Highlights of Spanish Astrophysics VI, eds. M. R. Zapatero Osorio, J. Gorgas, J. Maíz Apellániz, J. R. Pardo, \& A. Gil de Paz, Proc. IX Scientific Meeting of the EAS, 353

Schawinski, K., Thomas, D., Sarzi, M., et al. 2007, MNRAS, 382, 1415

Shaver, P. A., McGee, R. X., Newton, L. M., Danks, A. C., \& Pottasch, S. R. 1983, MNRAS, 204, 53

Singh, R., van de Ven, G., Jahnke, K., et al. 2013, A\&A, 558, A43

Swinbank, M., Sobral, D., Smail, I., et al. 2012, MNRAS, submitted [arXiv: 1209.1395]

Taylor, M. B. 2005, Astronomical Data Analysis Software and Systems XIV, eds. P. Shopbell, M. Britton, \& R. Ebert, ASP Conf. Ser., 347, 29

Thilker, D. A., Walterbos, R. A. M., Braun, R., \& Hoopes, C. G. 2002, AJ, 124, 3118

Trager, S. C., Faber, S. M., Worthey, G., \& González, J. J. 2000, AJ, 120, 165

Tremonti, C. A., Heckman, T. M., Kauffmann, G., et al. 2004, ApJ, 613, 898

van Zee, L., Salzer, J. J., Haynes, M. P., O’Donoghue, A. A., \& Balonek, T. J. 1998, AJ, 116, 2805

Vazdekis, A., Sánchez-Blázquez, P., Falcón-Barroso, J., et al. 2010, MNRAS, 404, 1639

Veilleux, S., \& Osterbrock, D. E. 1987, ApJS, 63, 295

Vergani, D., Epinat, B., Contini, T., et al. 2012, A\&A, 546, A118

Verheijen, M. A. W., Bershady, M. A., Andersen, D. R., et al. 2004, Astron. Nachr., 325, 151

Viironen, K., Sánchez, S. F., Marmol-Queraltó, E., et al. 2012, A\&A, 538, A144

Vila-Costas, M. B., \& Edmunds, M. G. 1992, MNRAS, 259, 121

Voges, E. S., \& Walterbos, R. A. M. 2006, ApJ, 644, L29

Webster, B. L., \& Smith, M. G. 1983, MNRAS, 204, 743

Yuan, T.-T., Kewley, L. J., \& Rich, J. 2013, ApJ, 767, 106

Zaritsky, D., Kennicutt, R. C., Jr., \& Huchra, J. P. 1994, ApJ, 420, 87

1 Centro Astronómico Hispano-Alemán, Calar Alto, (CSIC-MPG), C/Jesús Durbán Remón 2-2, 04004 Almería, Spain e-mail: dmast@caha.es

2 Instituto de Astrofísica de Andalucía (CSIC), Glorieta de la Astronomía s/n, 18008 Granada, Spain

3 Instituto Nacional de Astrofísica, Óptica y Electrónica, Luis E. Erro 1, 72840 Tonantzintla, Puebla, Mexico

4 Departamento de Física Teórica, Universidad Autónoma de Madrid, 28049 Madrid, Spain.

5 Leibniz Institute for Astrophysics Potsdam, an der Sternwarte 16, 14482 Potsdam, Germany

6 CEI Campus Moncloa, UCM-UPM, Departamento de Astrofísica y CC. de la Atmósfera, Facultad de CC. Físicas, Universidad Complutense de Madrid, Avda. Complutense s/n, 28040 Madrid, Spain

7 Institute of Astronomy, University of Cambridge, Madingley Road, Cambridge CB3 OHA, UK

8 CENTRA Centro Multidisciplinar de Astrofísica, Instituto Superior Técnico, Av. Rovisco Pais 1, 1049-001 Lisbon, Portugal

9 School of Physics and Astronomy, University of St Andrews, North Haugh, St Andrews KY16 9SS, UK

10 Instituto de Astrofísica de Canarias (IAC), Glorieta de la Astronomía S/N, 38206 La Laguna, S/C de Tenerife, Spain

11 Depto. Astrofísica, Universidad de La Laguna (ULL), 38206 La Laguna, Tenerife, Spain

12 Dep. Física Teórica y del Cosmos, Campus de Fuentenueva, Universidad de Granada, 18071 Granada, Spain

13 Centro de Astrofísica and Faculdade de Ciências, Universidade do Porto, rua das Estrelas, 4150-762 Porto, Portugal

14 Astronomisches Institut, Ruhr-Universität Bochum, Universitätsstr. 150, 44801 Bochum, Germany

15 RUB Research Department Plasmas with Complex Interactions, 44801 Bochum, Germany

16 University of Vienna, Türkenschanzstrasse 17, 1180 Vienna, Austria

17 Sydney Institute for Astronomy, School of Physics A28, University of Sydney, Sydney 2006 NSW, Australia

18 Australian Astronomical Observatory, PO Box 915, North Ryde NSW 1670, Australia

1930 Department of Physics and Astronomy, Macquarie University, North Ryde NSW 2109, Australia

20 Max Planck Institute for Astronomy, Königstuhl 17, 69117 Heidelberg, Germany 